\title{
The Spectrum of Proactive, Resilient Multi-Microgrid Scheduling: A Systematic Literature Review
}

\author{
Michael H. Spiegel $1, * \mathbb{E}$, Eric M. S. P. Veith ${ }^{2}(\mathbb{D}$ and Thomas I. Strasser $1,3(1)$ \\ 1 Electric Energy Systems-Center for Energy, AIT Austrian Institute of Technology, 1210 Vienna, Austria; \\ thomas.i.strasser@ieee.org \\ 2 Competence Cluster Applied Artificial Intelligence, R\&D Group Power Systems Intelligence, \\ OFFIS-Institute for Information Technology, 26121 Oldenburg, Germany; eric.veith@offis.de \\ 3 Institute of Mechanics and Mechatronics, Faculty of Mechanical and Industrial Engineering, TU Wien, \\ 1060 Vienna, Austria \\ * Correspondence: michael.spiegel@ait.ac.at
}

Received: 17 July 2020; Accepted: 22 August 2020; Published: 2 September 2020

\begin{abstract}
Multi-microgrids address the need for a resilient, sustainable, and cost-effective electricity supply by providing a coordinated operation of individual networks. Due to local generation, dynamic network topologies, and islanding capabilities of hosted microgrids or groups thereof, various new fault mitigation and optimization options emerge. However, with the great flexibility, new challenges such as complex failure modes that need to be considered for a resilient operation, appear. This work systematically reviews scheduling approaches which significantly influence the feasibility of mitigation options before a failure is encountered. An in-depth analysis of identified key contributions covers aspects such as the mathematical apparatus, failure models and validation to highlight the current methodical spectrum and to identify future perspectives. Despite the common optimization-based framework, a broad variety of scheduling approaches is revealed. However, none of the key contributions provides practical insights beyond lab validation and considerable effort is required until the approaches can show their full potential in practical implementations. It is expected that the great level of detail guides further research in improving and validating existing scheduling concepts as well as it, in the long run, aids engineers to choose the most suitable options regarding increasingly resilient power systems.
\end{abstract}

Keywords: asset scheduling; proactive scheduling; multi-microgrid; networked microgrid; microgrid; resilience; fault mitigation; systematic review

\section{Introduction}

Several decades ago, electrical networks called microgrids that can be both operated in a grid-connected and islanded mode were established. Such networks are often constructed to meet advanced power quality and reliability requirements which cannot be achieved by the main grid alone. Additional incentives include the economic interest in a tight integration of Renewable Energy Sources (RES), lowered purchase costs, as well as an increased efficiency by local generation [1]. Since the introduction of microgrids, several topics ranging from low-level voltage and frequency control up to high-level economic and reliable operation schedules of microgrid assets have been addressed [2,3].

A few years ago, the concept of multi-microgrids was introduced and has since attracted attention [4-6]. In most cases, multi-microgrids are defined as power systems, which incorporate multiple coordinated microgrids. Main driving forces in implementing multi-microgrids include resilience enhancements by supplying microgrid-external loads in case of contingencies as well as 
economic performance gains by sharing backup capacity and jointly optimizing normal operation. Following the diverse incentives and requirements, various multi-microgrid network topologies and coordination schemes [7] were presented. For instance, several microgrids can be connected via a common distribution system and a high-level controller may coordinate a jointly islanded operation of multiple connected microgrids in case the upstream grid fails. Furthermore, the distribution system may be split into multiple separate islands that are powered by connected microgrids within the island to circumvent distribution system faults. However, sharing backup capacity, in particular, requires a grid operation as one single or many parallel unconnected islands, which is not feasible in standard distribution systems.

As the local backup capacity is decreased and the extent of multi-microgrid setups is increased, the operation of a local microgrid may be affected by a drastically increased number of failures. Although for small independent microgrid installations it may be reasonable to consider main-grid failures only, additional failure modes such as line or generator tripping within a multi-microgrid gain in importance. Some techniques such as grid reconfiguration that mitigate the effect of various failures are already available. Nevertheless, most work on optimal, resilient microgrid and multi-microgrid operation only considers a limited set of failure modes and mitigation options [2]. Traditionally, the problems of optimal asset scheduling in normal operation and fault mitigation are discussed separately. However, several authors already started to incorporate advanced fault mitigation options and their side effects into their resilient scheduling approaches [8].

An exemplary multi-microgrid is depicted in Figure 1. Each of the three individual microgrids consists of various assets, such as controllable Distributed Energy Resources (DERs), energy storage units, volatile RES, and mixed-critical loads that may or must be supplied in case of contingencies. A deployed scheduling algorithm needs to control the microgrid assets before any contingency such that a resilient and cost-effective operation is achieved. A conventional scheduler, although it may consider the inherent stochasticity of loads, RES, and main-grid failures, may not consider the weak line L2-3. Even though the multi-microgrid can successfully tolerate main-grid outages, tripping L1-3 may cause an overload of the weak line L2-3 in case the power exchange between MG3 and the other microgrids exceeds the line capacity. To circumvent this situation, more generation needs to be scheduled at MG3, locally.

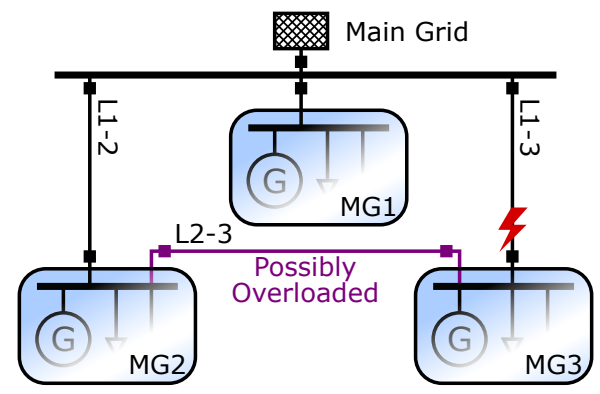

Figure 1. Exemplary multi-microgrid.

A broad variety of both fault mitigation techniques and targeted failure modes needs to be taken into account to ensure that microgrids and multi-microgrids are operated cost-effectively and yet at a guaranteed level of resilience. Such targeted failure modes include communication faults, as well as line- and generator tripping. Additionally, the inherent uncertainty of volatile RES generation, fluctuating loads and forecasting deviations need to be considered by a resilient schedule. The quality of the deployed models and probabilistic input assumptions calls for special attention, because inaccuracies may easily degrade the operation or increase the risk of faults.

Several competing methods which deal with the resilient operation and schedule various microgrid configurations are already available $[2,3,8]$. Similarly, various review papers which study multi-microgrid aspects were published [7,9]. Although some of them already introduce various resilience-related topics, none of them gives details on proactive asset scheduling that optimizes the 
normal operation while considering the influence on the system resilience. A sound overview of design decisions, implementation options, as well as the expected performance is needed to efficiently guide future research and to conduct the practical implementation of existing algorithms. For instance, several approaches to enhance resilience are summarized in [3], but an in-depth analysis of resilient multi-microgrid scheduling approaches, which also analyzes modeling and implementation details is still missing.

It is expected that a systematic study that covers aspects, such as modeling and validation approaches, will assist engineers and researchers likewise in selecting suitable methods for resilient multi-microgrid scheduling. This work addresses the concerns by first establishing a common view based on related literature in Section 2. The subsequent section specifically focuses on resilient pre-contingency scheduling in multi-microgrid setups. Section 3 presents the systematic methodology for selection and in-depth analysis of the identified key contributions. Results of the systematic review are stated and discussed in Section 4. Based on the review and contributions from related fields, an outlook on future research opportunities and engineering challenges is given in Section 5. Finally, the paper is concluded with Section 6.

\section{Work Related to a Resilient and Economic Multi-Microgrid Operation}

Substantial work has been contributed in related microgrid topics such as scheduling resources in microgrids, multi-microgrid forming and analyzing failure scenarios in power grids. Additionally, several contributions target the complexity of optimal asset scheduling in systems which face various sources of uncertainty (e.g., induced by volatile RES). At the same time, the approaches ensure that the microgrids can withstand certain failures $[2,10]$.

\subsection{Failure Modes and Resilience Metrics}

Despite the lack of a common definition of resilience in the context of power systems [8,11-13], some properties such as the ability to withstand and to recover from disruptive events are regularly associated with the term [8]. For instance, [11] that studies the definition of resilience in detail, defined the term with respect to an unexpected set of disturbances as "the system's ability to reduce the magnitude and duration of the disruption". The related term robustness is declared as "the ability of a system to cope with a given set of disturbances and maintain its functionality" [11]. A resilient system is associated with the ability of downgrading the performance while a robust one maintains the desired performance in the presence of potentially disruptive events. A review of 12 resilience definitions is given in [8]. The authors noted that in addition to restoration aspects, several definitions also include the avoidance of degraded states that others associate with robustness, only [13].

One attempt towards a common definition of power system resilience was made in [12] which relates resilience to various other terms such as reliability and robustness. Reliability, i.e., the probability of a functional system, reflects the performance under given conditions and over a long period of time, while resilience emphasizes the time-varying conditions in a contained time frame. The authors concluded that new metrics are needed to reflect resilience and presented a generalized framework to define such metrics. Similarly, a framework for resilience metrics is proposed in [13], which considers resilience as a function of time that reflects the recovery from a disruptive event. The framework was applied to assess the resilience of a road network.

Special attention must be drawn to the definition of considered failure modes. To secure the operation of conventional power systems, often the $(N-1)$ robustness criterion-which states that an operation strategy has to withstand the outage of any single system component-is applied [4]. Nevertheless, more detailed failure scenarios and other reliability indices may be used as well to secure the operation of microgrids and multi-microgrids. For instance, fault tree analysis, a method which is commonly used in risk assessment, was applied in [14] to identify critical components and to estimate the reliability of microgrids. 
To assess the reliability of isolated microgrids without the need for a detailed stochastic characterization of volatile energy sources, [15] uses easily available capacity factors to approximate various reliability metrics. Alternative assessment strategies include Monte-Carlo-based methods, which sample a large number of scenarios to approximate the joint distribution of all uncertainties [16]. Although these methods primarily target robustness and reliability aspects, they may also be incorporated into scheduling problems to improve the resilience of a particular schedule.

\subsection{Resilience-Aware Microgrid Scheduling}

A solution for the economic dispatch problem in single microgrids that ensures a stable islanded operation was presented in [17]. The authors considered the effects of the primary control strategy on the scheduling decision in detail, but only static security margins were used to reflect forecasting errors. To guarantee that critical loads can be supplied in islanded mode, Ref. [18] presented a scheduling method that is based on robust optimization. One deterministic worst-case scenario is found to define the resilient operation. An optimal normal operation schedule is revised in case it lacks sufficient online capacity for switching to an islanded operation. Hussain et al. [19] studied scheduling in multi-microgrids and proposed a robust optimization-based approach to incorporate inherent uncertainties. Although they also considered the islanded operation schedule, no detailed physical network model and no grid-reconfiguration options are included.

Some work also directly deploys stochastic optimization. For instance, in [20], a two-stage stochastic optimization approach that takes various network constraints and the required spinning reserve into account was presented. The expected cost under the presence of stochastic phenomena was optimized by mapping the problem into a deterministic linear optimization. Demand-response actions in a stochastic scheduling problem were studied in [21]. The presented approach also considers reserve requirements for compensating fluctuations.

\subsection{Multi-Microgrid Forming}

Conventional outage management systems are designed to automatically locate faults and restore healthy portions of a distribution grid [22] but an islanded operation is rarely considered. The role of microgrids in enhancing resilience was highlighted in [23], which describes the option that these microgrids provide surplus power to restore parts of the distribution grid. A particular method to control the supply of external loads was presented in [24] considering that the time loads can be expectedly supplied with available energy reserves.

A Mixed Integer Linear Programming (MILP) formulation of the grid partitioning problem, which forms each radial partition by a single generator, is additionally given in [25]. Supplied loads are maximized and switching operations are minimized in [26] by partitioning healthy but islanded sections of distribution networks into self-sustainable microgrids. Although some of the outlined approaches study both normal and emergency operation, the impact of grid-reconfiguration options on local reserve requirements in normal operation mode is not considered. Nevertheless, presented fault mitigation and reconfiguration options may be used to refine reserve estimation in asset scheduling problems.

\subsection{Resilience-Aware Multi-Microgrid Scheduling}

Some work specifically targeting resilience-aware scheduling in multi-microgrid environments is already available. To reduce the high share of dispatchable DERs, the concept of provisional microgrids, i.e., less critical microgrids that rely on other microgrids for islanding, was introduced [27]. Notably, an uncertainty-constrained optimal scheduling model that also includes islanding constraints is given. In particular, the concept and formulation of provisional microgrids may be used in the planned work as well. A risk-based model of optimal energy exchange scheduling between networked microgrids is given in [28]. Multiple strategies to deal with inherent risks connected to the stochastic nature of load 
and generation are presented and evaluated. The presented risk measure may be as well applied to manage risks in other multi-microgrid setups that cover an extended range of failure scenarios.

\subsection{Related Reviews}

Since the introduction of microgrids decades ago, a vast amount of work has been contributed [2]. Several authors already presented review papers which summarize the State-of-the-Art (SotA) and systematize various microgrid-related aspects $[2,3,7,9,10,29-41]$. Table 1 gives an overview of selected related reviews and their main topic. First developments and initial field test of microgrids were covered by [29-31], which describe several experimental sites and practical experiences. In [39], further developments and test sites with a strong focus on the United States are listed. Major challenges in including RESs into microgrids, such as scheduling under uncertainty, reliable and economic operation, as well as market-model designs are identified in [32]. The authors highlighted that microgrids can benefit from hierarchical control schemes allowing for a compromise between fully centralized and fully decentralized controls and summarized the SotA in the context of the three-layer scheme. A comprehensive review of various microgrid-related aspects including economics, protection, grid-supporting functions and clustered microgrids is given in [2]. Broad and condensed overviews of microgrids [36] and microgrid management systems [37,41] are also available. The application of multi-agent control in microgrids and microgrid clusters was specifically reviewed in $[34,35]$ studied differences in control architectures.

Table 1. Overview of related reviews.

\begin{tabular}{lll}
\hline Ref. & Publication Date & Main Topic \\
\hline$[29]$ & $2007-06-02$ & Microgrid-related research, development and demonstration effort \\
{$[30]$} & $2008-05-02$ & Testing experiences in experimental microgrids \\
{$[31]$} & $2010-10-01$ & Experimental and simulation-based microgrid test installations \\
{$[32]$} & $2014-04-20$ & Microgrid control strategies \\
{$[42]$} & $2015-05-12$ & Power system resilience \\
{$[2]$} & $2015-06-10$ & Broad review of microgrid-related topics \\
{$[10]$} & $2016-02-02$ & Optimization-based energy management in microgrids \\
{$[33]$} & $2016-09-16$ & DERs scheduling for microgrids and virtual power plants \\
{$[9]$} & $2017-06-30$ & Overview of multi-microgrids and available demonstration platforms \\
{$[34]$} & $2017-10-09$ & Distributed control and optimization of microgrids and multi-microgrids \\
{$[7]$} & $2017-12-22$ & Multi-microgrid architectures \\
{$[35]$} & $2018-03-22$ & Microgrid management system architectures \\
{$[36]$} & $2018-04-03$ & Recent microgrid-related developments and regulations \\
{$[37]$} & $2018-04-05$ & Microgrid energy management systems \\
{$[38]$} & $2018-06-11$ & Resilience of microgrids and multi-microgrids \\
{$[39]$} & $2018-06-18$ & Microgrid achievements in the United States \\
{$[40]$} & $2018-11-15$ & Networked microgrids \\
{$[8]$} & $2018-12-10$ & Impact of microgrids on power system resilience \\
{$[43]$} & $2018-12-13$ & Power system resilience \\
{$[3]$} & $2019-02-13$ & Resilience aspects in microgrids \\
{$[41]$} & $2019-03$ & Energy management in microgrids \\
{$[44]$} & $2019-12-09$ & Optimal planning and operation of islanded microgrids \\
\hline
\end{tabular}

In [10], a detailed review of energy-management approaches for microgrids is presented. The authors specifically listed related review work and practical implementations. Additionally, aspects such as objectives, constraints, solution approaches, as well as tools were reported. Although the work does not specifically evaluate resilience aspects such as failure modes and modeling assumptions, it is used as one basis for classifying optimization types and objectives. Similarly, [44] reviewed and categorized objectives, constraints and variables of optimization problems in islanded microgrids. The study focuses on islanded systems without considering multi-microgrid, resilience, and implementation-related aspects in detail, but results are used in the own research as another basis of classification and to provide a broad context. Scheduling approaches for microgrid and 
virtual power plant concepts are reviewed in [33]. Among other aspects, they addressed scheduling problems that are associated with reliability and stability issues and listed various reliability metrics. A comparative review of features such as supported DER types, type of formulation and solving methods is used as another basis to formulate a common feature set. Multi-microgrids or networked microgrids are addressed by $[9,40]$, who gave a broad overview of that topic and summarized several contributions related to planning and operation of multi-microgrids. A detailed study of multi-microgrid architectures and their impact on various aspects including costs, protection, as well as business models is given in [7]. One can observe that there are already some reviews targeting specific multi-microgrid and scheduling topics. However, resilience-relevant scheduling details such as modeled DERs and grid parameters as well as failure modes and effects are not covered in detail $[2,7,9,30,31,33-36,39-41,44]$.

Resilience in the broader context of power systems was studied by [42,43], but few details on resilient scheduling were given. In [8], a study on the definition of power system resilience is given and the strategies to increase power system resilience via microgrids are briefly discussed. Microgrid and multi-microgrid concepts that specifically focus on resilience aspects were reviewed in [38]. The authors introduced mechanisms for a resilient operation such as control strategies for an emergency operation, and briefly categorized common optimization terms. Although the categorizations are successfully used as a basis for own refinements, the link between pre-event operation and post-event fault mitigation techniques is weakly described. Significant details such as considered failure modes and validation approaches of pre-event scheduling algorithms are missing as well. Work presented in [3] specifically focuses on resilience-related aspects in microgrid operation. They covered a broad range of topics such as disaster modeling, outage management, and proactive scheduling. One research gap that was identified in [3] is the need for proactive scheduling approaches.

\subsection{Contributions of This Paper}

This paper refines findings of [3] and targets the identified research gap by systematically studying relevant technical details of work related to proactive, resilient multi-microgrid scheduling. It does so by applying a systematic review methodology that first assesses a large amount of candidate contributions and condenses a set of most relevant key contributions. A thoughtful analysis of the key contributions categorizes various details of the algorithms including modeling, optimization, validation and resilience aspects. This paper identifies the main design options that were exploited in resilient multi-microgrid scheduling approaches and highlights the development stage of these algorithms. It shows the wide variety of optimization-based contributions and illustrates that the work is on an early development stage that merely exceeds simulation-based validation. Given the detailed breakdown, this paper may be used to quickly identify related work and select suitable design options for upcoming research and engineering activities. The extensive outlook on research perspectives further discusses research gaps and related work that may be successfully applied in proactive resilient scheduling as well.

\section{Systematic Review Method}

As review studies always have a chance of giving biased results, the Preferred Reporting Items for Systematic Reviews and Meta-Analyses (PRISMA) statement [45] is used as a basis for the systematic review process. By following the recommendations, a reduction of bias and an increased transparency in the quality of the presented study is targeted. Figure 2 outlines the review procedure. At first, review candidates are identified and screened. Afterwards, eligible literature is iteratively reviewed. Finally, results from all reviewed papers are discussed and evaluated.

Due to the strong focus of the PRISMA methodology on medical studies, the process was slightly adapted to the needs of engineering studies. For instance, instead of focusing on achieved results and performance metrics, strong emphasis was put on the methodology such as modeling aspects and solution methods. Additionally, it was decided to merge the screening step that filters work according 
to the abstract and the eligibility test that accesses full-text articles in favor of a combined selection step that individually meets the review requirement to evaluate the selection criteria, i.e., full-test articles are accessed as soon as the selection criteria cannot be checked based on the abstract alone. Despite these modifications from the original PRISMA methodology, the overall process description was taken from [45].

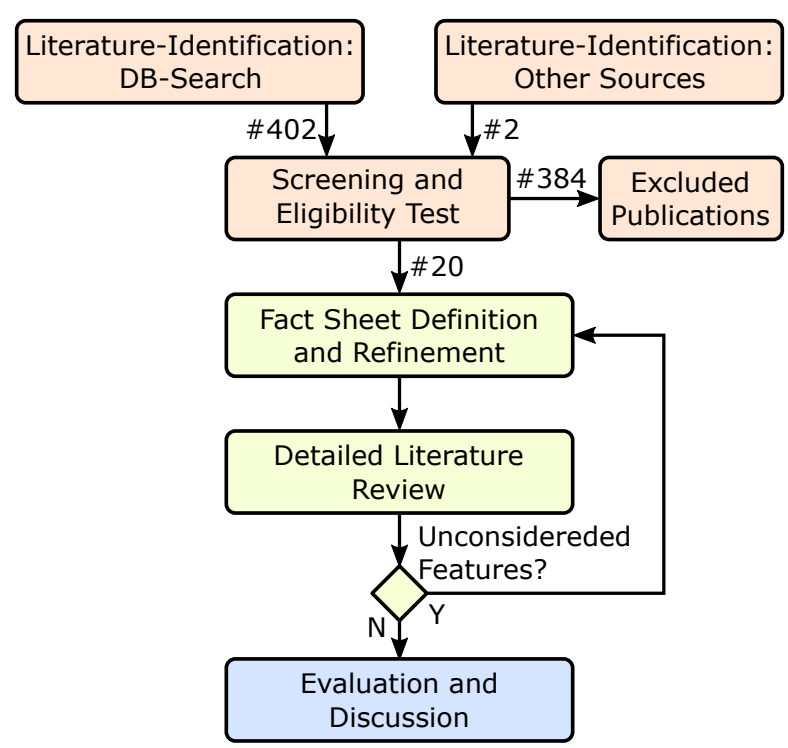

Figure 2. Review methodology workflow based on [45].

First, definitions and the research question are stated in Sections 3.1 and 3.2, respectively. Based on the study objectives, a broad literature screening was conducted. Section 3.3 describes the literature identification, screening and initial selection process in detail. For the detailed review of selected key contributions, a fact-sheet template was developed and in combination with the selection criteria, iteratively refined. Details on the fact sheet are presented in Section 3.4. The protocol including the identification steps and screened literature is published as supplementary material.

\subsection{Definitions}

Resilience is often used in the literature to characterize a system's capability of sustaining and recovering from hazardous impacts without an entire loss of functionality [8]. In opposition to other concepts such as robustness, which do not fully reflect a graceful degradation and service restoration, this publication defines the term resilience as "the ability to reduce the magnitude and/or duration of disruptive events" [12]. However, it must be emphasized that no common sense of resilience is established [11-13]. For instance, [13] defined resilience with respect to a delivery function as the ratio of recovery at a certain time to the loss at a disruptive event. An event is considered disruptive if and only if the magnitude of the delivery function is reduced. With respect to microgrids and the definition of [13], one can argue that a system is robust but not resilient, when no loss of load is encountered in case of a potentially disruptive event. An algorithm may be able to sustain potentially disruptive events without encountering a loss of load and consequently would be considered robust but not resilient with respect to that event. However, deploying that robust algorithm may still have the potential to increase the security of power supply in the presence of extreme events and failures. Furthermore, the strict definition may or may not include mitigation techniques, depending on their impact on the target metric.

In contrast to [13] and to mitigate problems of definition, the systematic review uses the term resilience in a broader context presented by [12]. This paper explicitly includes approaches which try to avoid power delivery failures of any kind. Nevertheless, considered algorithms may also permit a degraded performance in case of disruptive events. Hence, this paper refers to resilience-oriented 
multi-microgrid scheduling as "short-term resource planning, which is not restricted to avoiding outages entirely, but which also considers rapid recoverability in case of power shortages" [46]. Figure 3 illustrates the definition of resilience and the point in time, where normal and emergency mode scheduling decisions are taken.

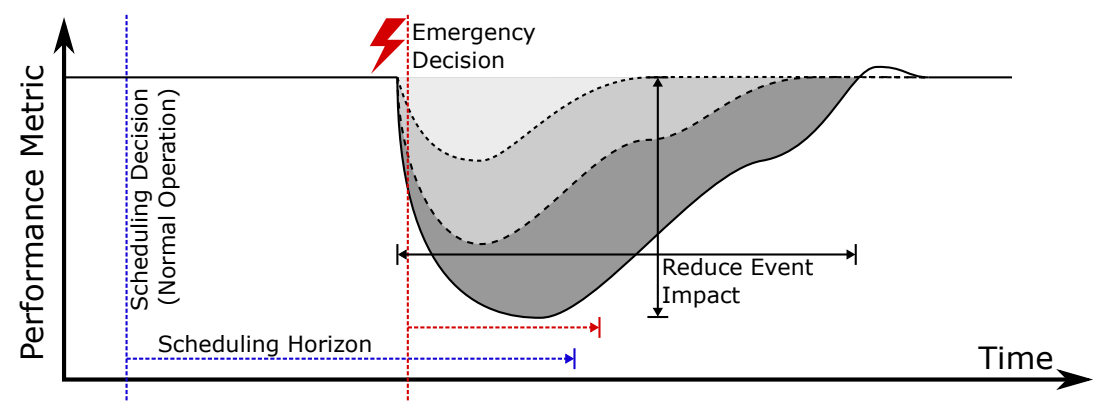

Figure 3. Resilient scheduling based on [12,13].

Similar to the definition of resilience, several definitions of microgrids do exist [32]. Some definitions include isolated microgrids which do not have a connection to a main grid and permanently operate in islanded mode. Since the work focuses on interconnected multi-microgrids, islanded grids are considered to be beyond the scope. Hence, microgrids are herein understood as electrical power systems that "can be operated in a non-autonomous way, if interconnected to the grid, or in an autonomous way, if disconnected from the main grid" [5] (p. 4).

\subsection{Research Questions}

The given review requires definition and assessment of a large amount of features that potentially impact the performance of scheduling algorithms. In order to contain the scope of the work and to select the most relevant aspects for review, the research question is explicitly defined, first. Starting from the motivation of achieving a resilient and economic operation of multi-microgrids and choosing suitable approaches for practical implementation and further research, the following main research questions emerges.

Which types of resilience-oriented scheduling functions for multi-microgrids do exist and how do they differ from one another?

To highlight the spectrum of resilient multi-microgrid scheduling functions, several sub-questions need to be answered. In particular, the following sets of sub-questions are used to guide the selection of extracted features.

1. Which aspects were modeled in SotA approaches? Such aspects include the type of DERs and their models, topological assumptions, failure modes, as well as the formulation of the objective functions. Additionally, sources and representations of uncertainty that are covered by presented models are addressed.

2. Which type of proactive actions and fault mitigation techniques are considered to increase resilience and efficiency? For instance, shiftable loads and controllable generation may be scheduled such that certain types of faults can be tolerated. Other actions may include grid-reconfiguration measures and in case of post-fault actions various islanding schemes [3].

3. How are these functions tested and evaluated? Which methodology is used to evaluate given approaches? Which input conditions (e.g., test networks, load and DER profiles) are used and do they differ in the degree of detail from the models used for optimization? Is there a common set of benchmarks? Which types of metrics are used to evaluate the performance of scheduling algorithms?

From a practical perspective, several design decisions need to be taken before implementing a resilient multi-microgrid scheduling approach. Figure 4 illustrates the main design decisions that are 
covered by the research question. The graphic divides the set of decisions into five categories such as modeling aspects and resilience features. Each design decision is linked to the other decisions. For instance, multi- and single-objective formulations must be solved differently.

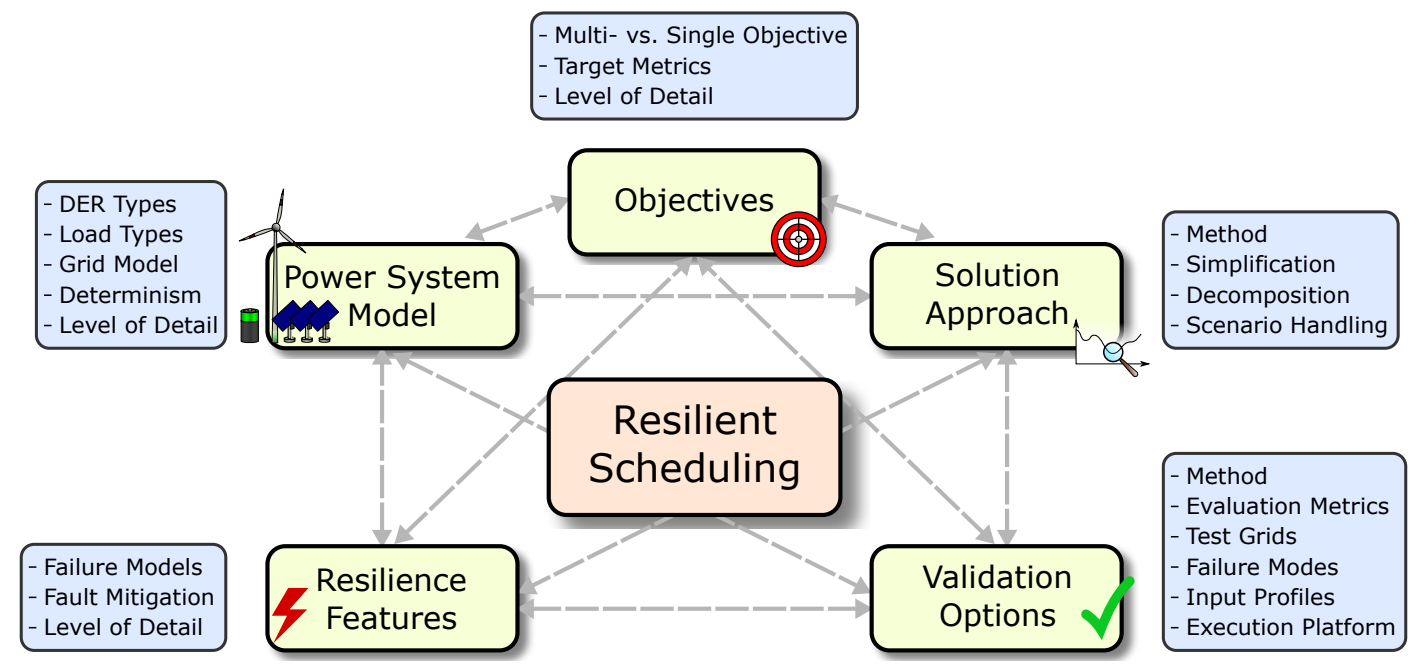

Figure 4. Resilient multi-microgrid scheduling: Design decisions.

\subsection{Literature Identification and Screening}

The primary literature was identified by several database searches. Due to the large coverage that includes numerous major databases and the subsequent manual selection process that tolerates less significant results, Google Scholar was selected as search engine. Since the relevance of displayed matches dropped considerably according to their ranking, a limit of 80 entries per search term was found to be appropriate. An initial search was conducted at the end of 2018 which was updated and extended in the first quarter of 2020. Further details including the list of search terms, matches and screening results can be found in the supplementary materials. Including several matches that were generated by multiple search terms, 404 publications were identified and screened for eligibility, in total. A list of criteria was defined to filter relevant publications that can be used to answer the research question. Each publication which was considered for the detailed review must fulfill the following criteria. Please note that a paper from the identification step can be rejected by multiple reasons. Consequently, the sum of rejections is bigger than the sum of rejected papers. No two papers which describe the very same approach were identified and no paper which describes multiple approaches is included. Hence, the number of papers corresponds to the number of studied approaches.

- Full-Text Availability: One of the basic formal criteria for including the paper in the systematic study is its availability. A paper was included if and only if the full content is available in English or German language. Six papers out of 404 were excluded due to their availability.

- Scheduling Algorithm Available: For further evaluation, a scheduling algorithm which covers essential technical details must be given. Scheduling algorithm is thereby understood as an algorithm which computes the set-points of controllable assets such as gensets, battery storage, and controllable loads in advance. Algorithms and papers which focus on long-term resource planning or placement, e.g., for optimizing strategic investment, and therefore not only consider set-points but also asset variations are considered to be out of scope. In the screening step, 140 papers were excluded because they do not describe a scheduling algorithm.

- Focus on Normal Operation: By definition, a microgrid or multi-microgrid is considered to be in normal operation if it is connected to the main grid. Hence, algorithms which solely cover an islanded or emergency operation were rejected for review. Additionally, if an approach focuses on resilience parameters such as critical load supply only, without considering operation metrics 
such as costs relevant in normal operation, it is not considered for review. In total, 75 papers were excluded because they focus on emergency operation only.

- Resilience Aspects Covered: To answer the research question on resilient scheduling, only approaches which tackle resilience aspects need to be covered. Work is herein considered in case one failure mode during normal operation is taken into account in a way that tolerance of this failure mode is ensured (i.e., robustness) or the impact of these failures is minimized (i.e., resilience). Such failure modes include (main-)grid failures, communication failures, or generation faults. An approach is not considered to be resilient scheduling, in case it does not actively shape resilience and only operates on static security margins without deriving them form the microgrid properties or validating them in an independent simulation. Such static margins include spinning reserve requirements are not actively adapted to the microgrid state. While screening, 98 papers were found to neglect resilience aspects considerably.

- Covered Technology: To guarantee a broad applicability of the reviewed approaches and to be able to compare several detailed aspects, a minimum set of modeled assets is defined [30,31]. In particular, each approach must at least be able to handle a schedulable energy storage, non-schedulable, stochastic loads, stochastic generation, and in accordance with the microgrid definition, an interruptible connection to the main grid. In particular, non-schedulable generation covers a wide range of RES which can be subsumed under this term [2]. In total, 46 papers do not cover the required technology and are therefore not considered for further review.

- MG Topology and Topological Constraints: A distribution system, which connects multiple, coordinated microgrids or which forms multiple, coordinated islands may not be able to handle every configuration without voltage and current violations [26]. It is, therefore, expected that network constraints such as topological constraints gain an increasing importance in multi-microgrids, compared to geographically contained single microgrids. Even if an algorithm does not actively alter the network topology, controls and scheduling decisions can have a considerable impact on a reliable operation [47].

Since the study focuses on a resilient operation of multi-microgrids, it is decided that included work must take topological constraints into account. In particular, power-flow limits and the topology of the microgrid or multi-microgrid must be taken into account. An algorithm which assumes a single-bus microgrid is not considered to be multi-microgrid scheduling, even if power transfer constraints to the main grid are modeled. From the initial set of identified publications, 31 papers do not consider network constraints at all and 15 papers only model a single-bus microgrid with limited transfer capabilities to the main grid. Similarly, four papers were excluded because they only model Alternating Current (AC)/Direct Current (DC) converter assets without considering transmission constraints within the network.

\subsection{Fact Sheet and Feature Extraction}

A detailed template was developed to guide the systematic review of selected papers and to answer the research questions given in Section 3.2. Based on related studies, a basic set of relevant features that should be extracted from each paper was recorded. The classification of optimization types such as MILP, and the classification of constraints including storage constraints were taken from [10]. The initial classification from [10] was further extended by the features of $[33,38,44]$ such as the additional DER and objective types.

The basic features from literature were substantially refined and new features which are needed to answer the research question were added. Results listed in $[10,33,44]$ were used to validate findings from this study. Although [10] lists the formulation of several objective functions in detail, it was decided to provide a categorized view, thereby easing the comparability of various approaches and the readability of the study. Hence, the fact-sheet template that defines recorded features was designed such that most of the features can be specified by assigning a category. For instance, it can be specified 
for each considered DER, whether it was modeled deterministically, indeterministically, stochastically, or not at all.

The fact sheet is structured in four feature groups. The first group covers model-related aspects such as details on DER, and in addition to related reviews, aspects related to the grid and load models. The optimization-related group covers the objectives and solution-related facts. One group specifically addresses resilience-related facts such as considered failure modes and fault mitigation techniques, and the last group lists various details on validation-related aspects. Although the overall structure was defined beforehand, the open-ended research questions such as the one on applied objective functions require an adaptive approach that allows the evaluation of features which were not considered beforehand. The fact-sheet template as well as reviewed facts were therefore iteratively refined as soon as new, relevant features were identified.

\section{Results and Discussion of the Systematic Review}

Based on the initial screening and eligibility test, the key contributions listed in Table 2 were selected for a detailed review. The first key contribution that leads towards resilient multi-microgrid scheduling dates back to September 2012. Although [48] already integrated dynamic grid-reconfiguration options into the resilient scheduling formulation, no dedicated multi-microgrid setup which potentially distributed ownership and independent islanding capabilities was addressed. A next step towards the integration of multiple independent microgrids was presented in [27]. The paper introduced the concept of provisional microgrids that use the grid-forming capabilities of a coupled independent microgrid in case of emergencies.

Table 2. Selected key contributions.

\begin{tabular}{|c|c|c|}
\hline Ref. & Publication Date & Main Topic \\
\hline [48] & 2012-09-28 & Integration of dynamic topology option into economic microgrid operation \\
\hline [49] & 2014-04-09 & Optimal scheduling of DER considering the risk of outages \\
\hline [27] & 2014-09-26 & Interaction of microgrids with and without independent islanding capabilities \\
\hline [50] & 2016-06-13 & Value of reconfigurable microgrids in integrating Electric Vehicles (EVs) \\
\hline [20] & 2016-08-10 & Resilient scheduling of microgrids affected by uncertainty \\
\hline [51] & 2016-08-26 & Multi-objective scheduling of microgrids considering normal operation costs \\
\hline [52] & 2016-10-15 & $\begin{array}{l}\text { and the risk of load curtailment } \\
\text { Microgrid scheduling considering operating costs, emissions, and reserve } \\
\text { requirements }\end{array}$ \\
\hline [53] & 2017-08-17 & Robust formulation of the optimal proactive scheduling problem for microgrids \\
\hline [28] & 2017-12-13 & Risk-based strategies for multi-microgrid scheduling considering stochastic RESs \\
\hline [54] & $2018-02-16$ & Optimal scheduling for hybrid AC/DC multi-microgrids \\
\hline [55] & 2018-02-20 & $\begin{array}{l}\text { Integration of Demand Response (DR) programs and grid reconfiguration into } \\
\text { microgrid asset scheduling }\end{array}$ \\
\hline [56] & 2018-03-26 & Resilient asset scheduling in reconfigurable microgrids \\
\hline [57] & 2018-05-11 & Security-constrained dispatch for microgrids using multi-objective optimization \\
\hline [58] & 2018-08-17 & Resiliency enhancements by optional scheduling of networked microgrids \\
\hline [59] & 2018-12-28 & Impact of scheduling discrepancies on interconnected microgrids \\
\hline [60] & 2019-04-18 & $\begin{array}{l}\text { Microgrid scheduling combining flexible time frame DER scheduling and single } \\
\text { time interval-based optimal dispatch }\end{array}$ \\
\hline [61] & 2019-07-02 & Resilient scheduling of networked multi-microgrids using a three-stage approach \\
\hline [62] & 2019-08-07 & Proactive, resilient scheduling of interconnected microgrids \\
\hline [63] & 2019-08-16 & $\begin{array}{l}\text { Distributed energy management of interconnected microgrids considering } \\
\text { adversarial actions }\end{array}$ \\
\hline [64] & 2020-01-06 & Optimal, resilient operation of dynamic multi-microgrids \\
\hline
\end{tabular}

The first selected key contribution that specifically addresses multi-microgrid setups is [28] that was published at the end of 2017. Evidently, the authors did not present the first multi-microgrid setup, ever $[7,28]$, but a significant contribution was made in considering resilience aspects in multi-microgrid scheduling. Since then, several authors also directly considered multi-microgrid setups in resilient scheduling formulations $[54,58,61-64]$. Although only in very recent years identified key contributions 
show a tendency to explicitly cover multi-microgrids, literature that focuses on independent microgrids may be applicable in the extended setting as well.

None of the eligibility criteria directly states that a scheduling approach needs to be explicitly formulated as an optimization problem. A scheduling algorithm may be as well formulated as some simple heuristic rules that eliminate the need for an online optimization procedure [37]. For instance, [65] presented a heuristic EV charging strategy that does not require any optimization steps. The key contributions show a wide variety of different models, resilience features, and optimization approaches including heuristic optimization. However, every selected publication follows an optimization-based framework.

\subsection{Selected Literature}

The screening of identified literature and the eligibility test were performed in an interleaved process. In case the paper abstracts did not provide sufficient information to classify the work with certainty, a more detailed screening was conducted. Although the authors tried to define the eligibility criteria as precisely as possible, some border cases still had to be handled. For instance, the early work [17] that studies a scheduling problem of distributed generators considering main-grid faults was excluded due to the fact that energy storage units were insufficiently tackled. Although the work gives valuable insights into reserve commitment, Electrical Energy Storages (EESs) such as battery storage systems are considered to be a canonical part of future multi-microgrids. Similarly, the work in [21] that specifically tackles reserve scheduling was excluded due to the limited consideration of failure modes. Judging from the formulation, managed reserves are used to balance RES and load fluctuations only, without considering failure scenarios such as main-grid outages and their mitigation. Although the paper provides valuable insights into the effects of DR programs, it was excluded from the list of key contributions because it violates the resilience criteria.

No obvious classification was found for [57] either, which describes a security-constrained optimal dispatch approach. Although the stochastic behavior of RESs and loads is not explicitly taken into account, terms in one objective function penalize main-grid transfer and storage usage. Hence, available reserves that are used to balance fluctuations are systematically maximized and the paper is considered to be a key contribution. A similar border case is given by [27] that introduces the concept of provisional microgrids. The provisional microgrid is modeled as a single bus only, but the topological constraint that provisional microgrids cannot be islanded independently of a coupled microgrid, is given. The connection to the coupled microgrid including its power-flow limitations is also modeled in addition to the main-grid connection. Despite the single-bus limitation, some topological constraints that supplement the main-grid connection are provided and the paper was selected.

In [66], a proactive scheduling approach of hybrid AC/DC microgrids was described. The microgrid was modeled by two buses, one connecting the AC assets and the main grid as well as one connecting the DC facilities. Since the paper focuses on independent hybrid microgrids, no topological constraints, despite one interlinking converter that couples both buses and main-grid transfer constraints, were given. Unlike $[27,66]$ does not study the scheduling approach in the context of other microgrids and was therefore not selected as a key contribution. A similar border case was given by [67] that provides valuable insights into the resilient operation of networked hybrid AC/DC microgrids. However, the publication does not include any topological constraints such as line capacity constraints and was therefore not selected as a key contribution either.

\subsection{Modeling Approaches and Modeled Assets}

Each of the key contributions models a set of controllable and uncontrollable microgrid assets. Some of them use generic models such as generic unschedulable DERs (e.g., [27,52,64]) while others specifically model the properties of some assets such as Wind Turbines (WTs) including their turbine characteristics (e.g., [28,58]). Table 3 summarizes modeled DER parameters and classifies common DER constraints. In case only a generic model is considered, no specific model is listed although the 
specific asset may be covered by the generic model as well. Some publications such as [64] explicitly use the term battery energy storage but no significant difference to the formulation of generic EESs including $[28,62]$ was found. Hence, these battery energy storage models were classified as generic EESs as well.

Most of the key contributions stick to generic DER and EES models without specifically focusing on more specific assets. Only one paper also considers Combined Heat and Power (CHP) plants and the thermal needs of a microgrid [62]. Similarly, one out of 20 papers specifically targets Micro Turbines (MTs) [28] and two contributions take EVs into account [20,50]. None of the contributions directly considered hydro turbines, pumped storage, fuel cells or hydrogen storage. Although there are papers such as [68] that consider hydrogen infrastructure in scheduling problems, none of the screened work meets all eligibility criteria for resilient multi-microgrid scheduling. In particular, [68] provided valuable insights into the operation of reconfigurable microgrids considering reliability and degradation effects caused by asset use. However, due to the lack of resilience features such as islanding, it was excluded from the detailed analysis.

In addition to the principal DER classes, Table 3 also indicates the level of determinism. Deterministic (D) formulations that do not incorporate any uncertainty, stochastic (St) models that include probabilistic information, and indeterministic (ID) formulations that do not use any probabilistic assumptions on the distribution of uncertain values are explicitly highlighted. Figure 5 illustrates the levels of determinism via a single deterministic curve, and two ensembles of curves that are defined by indeterministic intervals and probability density functions, respectively. One can observe that all generic controllable assets, i.e., EESs and DERs are modeled in a deterministic fashion. Intuitively, this makes sense because the low-level control of most controllable assets will ensure a proper operation according to the set-points. However, some publications such as [69] that considers frequent but random outages of a fuel cell in a scheduling formulation, also report stochastic phenomena of controllable assets.

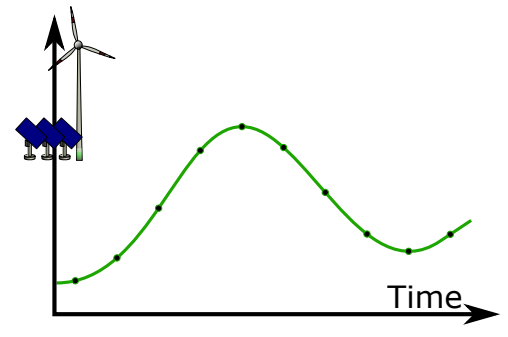

(a) Purely deterministic

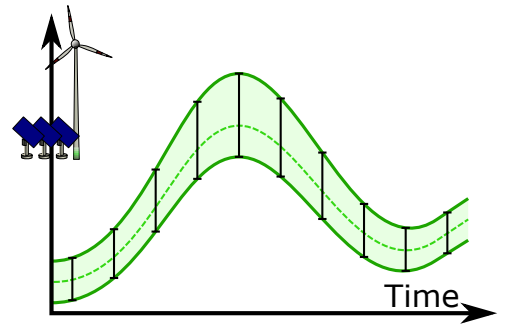

(b) Indeterministic, but not probabilistic

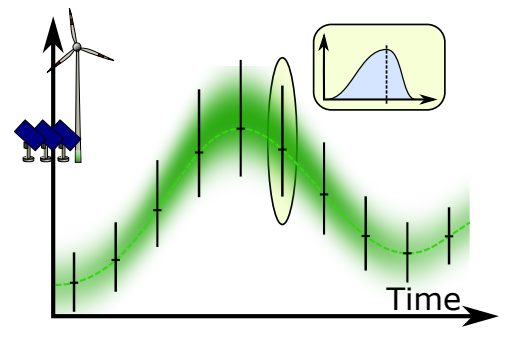

(c) Stochastic, including probabilistic information

Figure 5. Observed model and input types.

Although EVs that actively participate in a microgrid can be controlled while they are plugged in, the user behavior, in particular departure and arrival time, as well as State of Charge (SoC) requirements, is highly uncertain $[20,50]$. Both key contributions that consider EVs presented a stochastic EV model. However, also deterministic simplifications of driving patterns can be observed in some scheduling approaches [67].

For unscheduled DERs, most key contributions use a stochastic or robust formulation without purely relying on a forecast and optimizing security margins. Although for generic unscheduled DERs several authors including $[27,53,54,62]$ chose to follow an indeterministic formulation, most of the contributions that tackle the specifics of uncontrollable or partly controllable generation such as Photovoltaics (PV) and wind power plants, deploy stochastic asset models. Most of the specific asset models describe the conversion of the primary energy source, into electric energy [28,58]. For solar irradiation and wind speed, detailed studies on their distribution are available [70-72] and used in scheduling problems [28,58]. However, for an indeterministic robust model, the input 
distribution reduces to a set [19] that is often generalized to the set of expected unscheduled power generation $[53,54,62]$.

Diverse levels of detail are found for the DER models and are briefly summarized in Table 3. Every key contribution considered active power generation limits for the controllable assets. However, other limitations such as reactive and apparent power constraints are far less common. For instance, only eight out of 20 key contributions including $[53,56,62,64]$ explicitly considered reactive or apparent power constraints. For one publication, [49], not enough details were given to clearly categorize the constraint. In addition to various power constraints, 12 publications such as $[27,51,60]$ also include ramping constraints that limit the rate of change of the generated power. In order to avoid frequent start-up operations, eight key contributions (e.g., [28,52,58]) considered minimum up- and down-time constraints.

The common EES is modeled as a finite power and capacity constraint storage that accumulates the charged and discharged energy into a time-dependent SoC. In particular, only three key contributions did not constrain the energy that can be fed into or drawn from the energy storage $[28,49,55]$. Eighteen contributions model a constant storage efficiency that limits the energy that can be discharged. Although most of them assume that, like in $[20,64]$, losses are encountered while charging and discharging only, one contribution also considers self-discharge of the energy storage unit [63]. None of the key contributions modeled the storage efficiency as a non-linear relationship of charging/discharging power or the SoC. However, one paper considers the non-linear charging curve of EVs by modeling the maximum charging and discharging power with respect to the current SoC [20]. A minority of five contributions constrained the minimum charging- and discharging time of an EES [27,52], but most of the contributions did not include any constraints to limit the battery degradation.

In addition to DER types and parameters, Table 3 also lists the implemented load models. One can see that all the papers consider constant power loads, while only one paper considers voltage-dependent loads [20]. One type of assets that is commonly found in microgrids is sheddable loads as discussed in [53,54]. To differentiate sheddable loads from loads that may be unserved by accident, only loads that can be controlled by the scheduling approach are considered. In case a load is interrupted because, for instance, line protection trips that load is not considered to be sheddable load. In addition to loads, that can be interrupted based on a signal from the microgrid controller, shiftable loads that can be deferred [27,54,56,58] and price-based DR programs [55] are modeled. However, these load types are only considered in a minority of five papers.

Similar to generic unscheduled DERs, most contributions consider load uncertainties via indeterministic (e.g., [20,27]) or stochastic (like in [58,59]) models. In contrast, only three out of 15 papers consider an uncertain amount of sheddable loads as well $[53,61,64]$. Similar observations can be made for price-based DR loads and shiftable loads as well. No contribution was found that considers stochastic effects in the latter two load types.

Table 4 lists the evaluated features regarding the grid model and Figure 6 illustrates the listed model types. The model itself is categorized into several different types of formulations. In the simplest case, the topology is reduced to one single bus and only energy balances are taken into account. As described in Section 4.1, there is only a single paper that uses a single-bus model and that still meets the eligibility criteria of resilient multi-microgrid scheduling. To cover topological constraints without requiring a detailed physical model, three publications use arbitrary connection graphs $[54,58,63]$ and one publication restricts that graph to a star topology of microgrids that are connected via a distribution system [61]. For each node in the graph model, a power balance equation is stated and the power that is transferred from or to an adjacent grid can be constrained. However, power flows that result from the physical properties of the network and that may not be fully controllable, are beyond the scope of the model. 


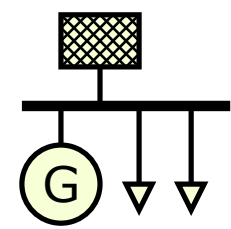

(a) Single bus

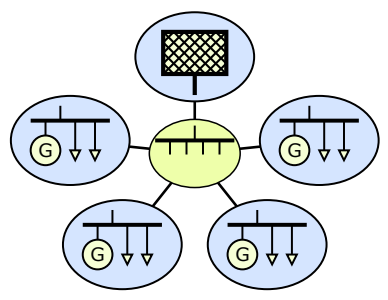

(c) Star

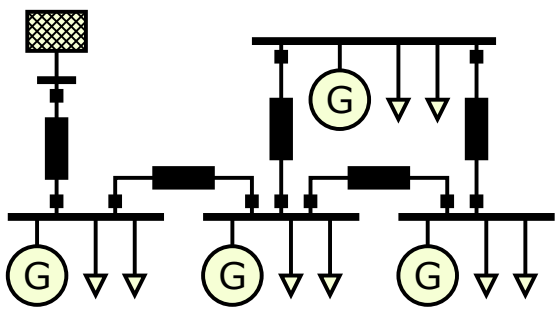

(b) Impedance

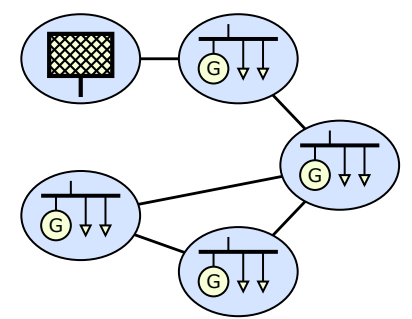

(d) Connection graph

Figure 6. Observed types of grid models.

One key publication [59] extended the basic graph model by considering the efficiencies of power lines and the resulting power losses. However, no detailed model that covers the impact of reactive power flows on the line efficiencies was given. To cover the power flows as they appear by the physical properties of the system, several contributions formulated the grid model as steady-state power-flow model based on the line impedance (e.g. $[48,55,57])$. Despite the common framework of using a steady-state power-flow model, various differences in the particular formulations can be observed. Some publications directly model the power-flow equations as non-linear relationship between the voltage magnitudes and angle differences of connected buses [55]. Others directly present a linearized version [48] or include a linearization procedure [56]. Notably, some publications indirectly reference the power-flow model by using an external solver in their scheduling approach $[51,57,60]$, but the majority explicitly states the power system model.

Nearly all publications that use impedance-based models also stated voltage constraints (e.g., [52,56,62]). Only [48] did not explicitly constrain the bus voltage. Hence, one additional value of the impedance-based model beyond realistic power flows is the containment of voltage problems caused by improper scheduling. In contrast, more detailed transient models that allow the assessment of the microgrid in switching or failure states are only used by [57]. Although the publication demonstrates the impact of scheduling on the short-circuit performance in terms of frequency response and voltage transients, scheduling models are mostly concerned with steady-state phenomena.

Another set of a commonly considered constraints are line capacity constraints (e.g., [48,56,59]). Only three key contributions did not consider the limited capacity of distribution lines. However, there is not a single formulation to address the line capacity given. Observed constraints include steady-state active [63], reactive [62], and apparent power-flow [50] constraints, as well as steady-state current limitations [60]. A majority of 13 key publications including [27,48,54] model main-grid transfer via a single point of common coupling. However, some publications such as [62] explicitly consider multiple connections to one or several utility grids as well [7]. Similar to the power line constraints, main-grid transfer is often restricted by active [20], reactive [48], and apparent [53] power constraints. 
Table 3. Modeled load and DER types ( $\checkmark$ : Implemented, ?: Not reported, St: Stochastic, D: Deterministic, ID: Indeterministic)

\begin{tabular}{|c|c|c|c|c|c|c|c|c|c|c|c|c|c|c|c|c|c|c|c|c|c|c|c|}
\hline & DER & ypes & & & & & & & & DE & $\mathbf{R} \mathbf{P a}$ & ame & & & & & & & Load T & ypes & & & \\
\hline & 3 & $\vec{c}$ & 苞 & 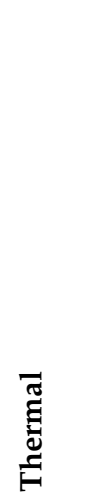 & $\sum_{i=1}^{\infty}$ & $\stackrel{\infty}{\dot{E}}$ & 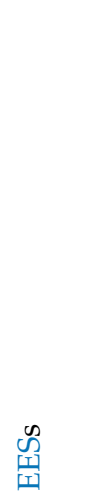 & 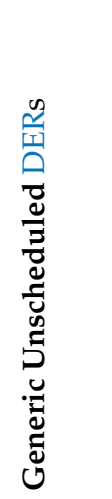 & 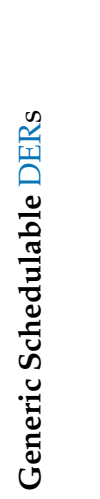 & 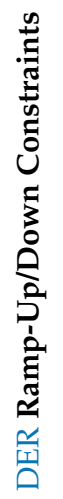 & 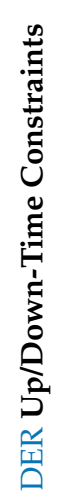 & 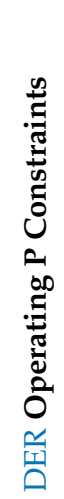 & 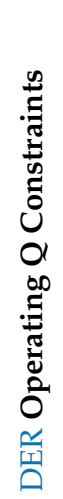 & 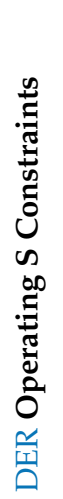 & 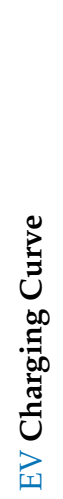 & 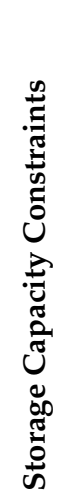 & 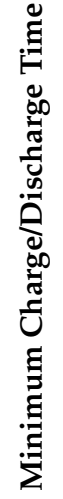 & 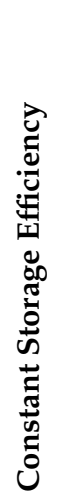 & 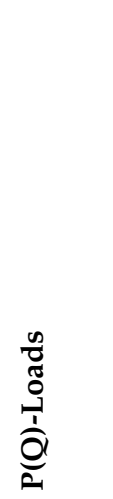 & 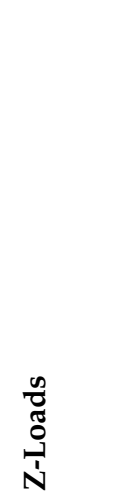 & 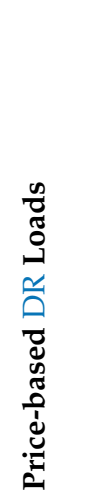 & 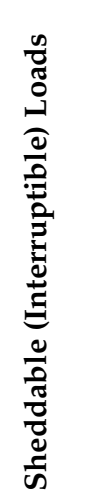 & 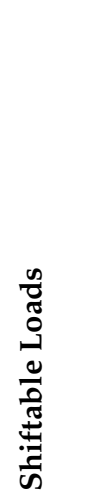 \\
\hline \multicolumn{24}{|l|}{ Ref. } \\
\hline [48] & & & & & & & $\checkmark(D)$ & $\checkmark(\mathrm{St})$ & $\checkmark(D)$ & & & $\checkmark$ & $\checkmark$ & & & $\checkmark$ & & $\checkmark$ & $\checkmark(\mathrm{St})$ & & & $\sqrt{ }(\mathrm{D})$ & \\
\hline [49] & $\sqrt{ }(\mathrm{D})$ & $\boldsymbol{V}(\mathrm{D})$ & & & & & $\sqrt{ }(\mathrm{D})$ & $\sqrt{ }(\mathrm{D})$ & $\sqrt{ }(\mathrm{D})$ & & & $\checkmark$ & & $?$ & & & & & $\sqrt{ }(\mathrm{D})$ & & & $\boldsymbol{V}(\mathrm{D})$ & \\
\hline [27] & & & & & & & $\sqrt{ }(\mathrm{D})$ & $\checkmark($ ID) & $\sqrt{ }(\mathrm{D})$ & $\checkmark$ & $\checkmark$ & $\checkmark$ & & & & $\checkmark$ & $\checkmark$ & $\checkmark$ & $\checkmark($ ID) & & & $\boldsymbol{V}(\mathrm{D})$ & $\sqrt{ }(\mathrm{D})$ \\
\hline [50] & & & & & $\checkmark(\mathrm{St})$ & & & $\mathcal{J}(\mathrm{St})$ & $\boldsymbol{S}(\mathrm{D})$ & & & $\checkmark$ & & & & $\checkmark$ & & $\checkmark$ & $\mathcal{J}(\mathrm{St})$ & & & & \\
\hline [20] & $\mathcal{J}(\mathrm{St})$ & & & & $\checkmark(\mathrm{St})$ & & $\boldsymbol{S}(\mathrm{D})$ & & $\boldsymbol{S}(\mathrm{D})$ & & & $\checkmark$ & $\checkmark$ & & $\checkmark$ & $\checkmark$ & & $\checkmark$ & $\sqrt{ }(\mathrm{ID})$ & $\boldsymbol{J}(\mathrm{ID})$ & & $\boldsymbol{J}(\mathrm{D})$ & \\
\hline [51] & & & & & & & $\sqrt{ }(\mathrm{D})$ & $\sqrt{ }(\mathrm{St})$ & $\sqrt{ }(\mathrm{D})$ & $\checkmark$ & & $\checkmark$ & & & & $\checkmark$ & & $\checkmark$ & $\mathcal{V}(\mathrm{St})$ & & & $\sqrt{ }(\mathrm{D})$ & \\
\hline [52] & & & & & & & $\checkmark(D)$ & $\sqrt{ }(\mathrm{St})$ & $\checkmark(D)$ & $\checkmark$ & $\checkmark$ & $\checkmark$ & $\checkmark$ & & & $\checkmark$ & $\checkmark$ & $\checkmark$ & $\sqrt{ }(\mathrm{St})$ & & & & \\
\hline [53] & & & & & & & $\checkmark(\mathrm{D})$ & $\checkmark(\mathrm{ID})$ & $\boldsymbol{S}(\mathrm{D})$ & $\checkmark$ & $\checkmark$ & $\checkmark$ & & $\checkmark$ & & $\checkmark$ & & $\checkmark$ & $\checkmark(\mathrm{ID})$ & & & $\boldsymbol{J}(\mathrm{ID})$ & \\
\hline [28] & $\mathcal{J}(\mathrm{St})$ & $\mathcal{J}(\mathrm{St})$ & & & & $\boldsymbol{S}(\mathrm{D})$ & $\sqrt{ }(\mathrm{D})$ & & & $\checkmark$ & $\checkmark$ & $\checkmark$ & & & & & $\checkmark$ & $\checkmark$ & $\mathcal{J}(\mathrm{St})$ & & & & \\
\hline [54] & & & & & & & $\sqrt{ }(\mathrm{D})$ & $\boldsymbol{J}(\mathrm{ID})$ & $\boldsymbol{}(\mathrm{D})$ & $\checkmark$ & $\checkmark$ & $\checkmark$ & & & & $\checkmark$ & & $\checkmark$ & $\sqrt{ }(\mathrm{ID})$ & & & $\sqrt{ }(\mathrm{D})$ & $\boldsymbol{J}(\mathrm{D})$ \\
\hline [55] & $\sqrt{ }(\mathrm{St})$ & $\mathcal{J}(\mathrm{St})$ & & & & & $\sqrt{ }(\mathrm{D})$ & & $\sqrt{ }(\mathrm{D})$ & $\checkmark$ & & $\checkmark$ & & & & & & $\checkmark$ & $\checkmark(\mathrm{D})$ & & $\sqrt{ }(\mathrm{D})$ & & \\
\hline [56] & & & & & & & $\checkmark(D)$ & $\sqrt{ }(\mathrm{St})$ & $\checkmark(D)$ & $\checkmark$ & $\checkmark$ & $\checkmark$ & $\checkmark$ & & & $\checkmark$ & $\checkmark$ & $\checkmark$ & $\mathcal{V}(\mathrm{St})$ & & & $\boldsymbol{J}(\mathrm{D})$ & $\mathcal{J}(\mathrm{D})$ \\
\hline [57] & & & & & & & $\checkmark(D)$ & $\sqrt{ }(\mathrm{D})$ & $\checkmark(D)$ & & & $\checkmark$ & & & & $\checkmark$ & & $\checkmark$ & $\checkmark(D)$ & $?$ & & $\mathcal{J}(\mathrm{D})$ & \\
\hline [58] & $\mathcal{J}(\mathrm{St})$ & $\mathcal{J}(\mathrm{St})$ & & & & & $\sqrt{ }(\mathrm{D})$ & $\sqrt{ }(\mathrm{St})$ & $\boldsymbol{S}(\mathrm{D})$ & $\checkmark$ & $\checkmark$ & $\checkmark$ & & & & $\checkmark$ & $\checkmark$ & & $\mathcal{J}(\mathrm{St})$ & & & $\sqrt{ }(\mathrm{D})$ & $\boldsymbol{J}(\mathrm{D})$ \\
\hline [59] & & & & & & & $\sqrt{ }(\mathrm{D})$ & $\sqrt{ }(\mathrm{D})$ & $\sqrt{ }(\mathrm{D})$ & & & $\checkmark$ & & & & $\checkmark$ & & $\checkmark$ & $\boldsymbol{V}(\mathrm{St})$ & & & $\boldsymbol{V}(\mathrm{D})$ & \\
\hline [60] & & & & & & & $\checkmark(D)$ & $\checkmark(\mathrm{D})$ & $\checkmark(D)$ & $\checkmark$ & $\checkmark$ & $\checkmark$ & $\checkmark$ & & & $\checkmark$ & & $\checkmark$ & $\checkmark(\mathrm{D})$ & & & $\checkmark(\mathrm{D})$ & \\
\hline [61] & $\checkmark(\mathrm{St})$ & & & & & & $\checkmark(D)$ & & $\sqrt{ }(\mathrm{D})$ & & & $\checkmark$ & & & & $\checkmark$ & & $\checkmark$ & $\mathcal{V}(\mathrm{St})$ & & & $\mathcal{J}(\mathrm{St})$ & \\
\hline [62] & & & $\boldsymbol{V}(\mathrm{ID})$ & $\checkmark(D)$ & & & $\sqrt{ }(\mathrm{D})$ & $\sqrt{ }(\mathrm{ID})$ & & $\checkmark$ & & $\checkmark$ & $\checkmark$ & & & $\checkmark$ & & $\checkmark$ & $\sqrt{ }(\mathrm{ID})$ & & & $\boldsymbol{V}(\mathrm{D})$ & \\
\hline [63] & & & & & & & $\sqrt{ }(\mathrm{D})$ & $\mathcal{V}(\mathrm{St})$ & $\boldsymbol{J}(\mathrm{D})$ & & & $\checkmark$ & & & & $\checkmark$ & & $\checkmark$ & $\mathcal{S}(\mathrm{St})$ & & & & \\
\hline [64] & & & & & & & $\mathcal{S}(\mathrm{D})$ & $\mathcal{V}(\mathrm{St})$ & $\mathcal{S}(\mathrm{D})$ & $\checkmark$ & & $\checkmark$ & $\checkmark$ & & & $\checkmark$ & & $\checkmark$ & $\mathcal{J}(\mathrm{St})$ & & & $\mathcal{J}(\mathrm{St})$ & \\
\hline
\end{tabular}


Table 4. Grid model ( $\checkmark$ : Implemented, ?: Not reported).

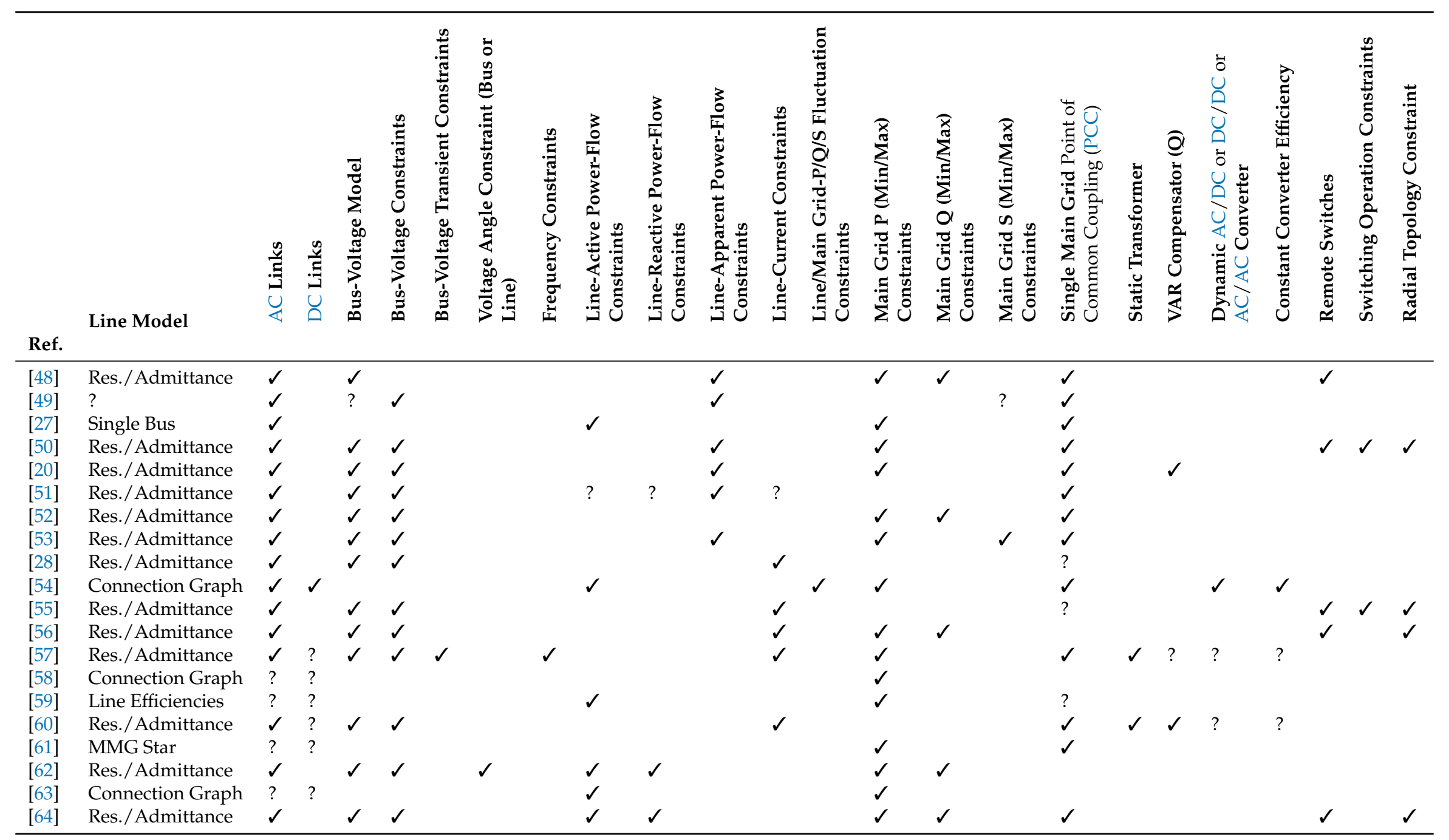


Although most of the key contributions focus on static grid models that consist of lines that connect DERs, loads and the main grid, few also consider controllable assets such as VAR compensators [20] and dynamic converters to control power flows [54]. Similarly, static transformer models are reported by two contributions only $[57,60]$. Five papers considered remote switching capabilities to dynamically alter the topology of a microgrid (e.g., [48,55]). Only one of these contributions, [48], considered meshed topologies. Every other publication that included dynamic topologies formulated constraints that always guarantee a radial topology $[50,55]$. Although some approaches including $[57,60]$ may directly support other grid assets such as tap changer transformers as well, none of the contributions reported their application.

A similar set of models and constraints can also be found in other related domains such as islanded microgrids [44]. In particular, DER output, ramping, power flow and balance constraints appear most frequently in microgrid operation and panning problems [10,33,44]. However, other constraints such as financial budgets and placement options are primarily found in planning problems. Although voltage and frequency control strategies may have a significant impact on the feasibility of mitigation techniques [57], few key contributions included detailed frequency constraints. Voltage constraints, in contrast, are far more often found in resilient normal operating problems. Considering recent publications on islanded microgrids, both voltage and frequency constraints are frequently found [44].

\subsection{Optimization Objective and Methods}

The typical resilient scheduling approach such as $[20,48,56]$ sketches a central controller that uses a mathematical solver such as a MILP solver to obtain the optimal schedule with respect to a single-objective function. However, there are various notable exceptions that follow a different approach. Table 5 summarizes the results regarding used optimization procedures. In addition to mathematical approaches that usually pass the problem on to a MILP solver, some heuristic optimization techniques such as genetic algorithms [28,51], Particle Swarm Optimization (PSO) [52,64], Imperialist Competitive Algorithm (ICA) [49], Clonal Selection Algorithm (CSA) [50], Exchange Market Algorithm (EMA) [55], and Pareto Concavity Elimination Transformation (PaCcET) [57] can be found [73]. Two contributions demonstrated an alternative to the central control paradigm in which one controller gathers all information and decides on the next schedule [54,63]. Publication [54] demonstrated a hierarchical architecture where single independent microgrid controllers are coordinated by a central supply-level controller. A fully distributed approach is presented by [63] which does not need a central entity at all. Figure 7 gives an overview of all three control paradigms and shows the controllers as well as the main points of interaction.

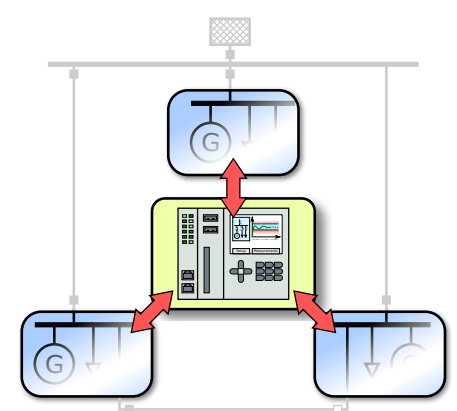

(a) Central

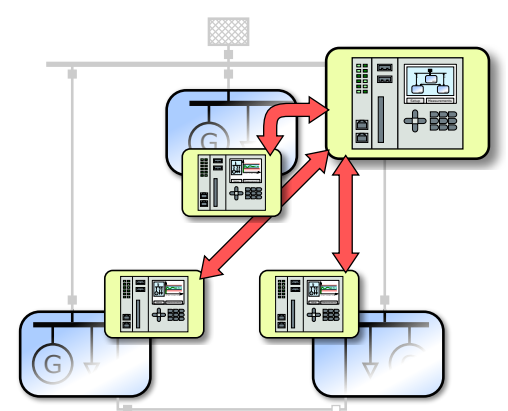

(b) Hierarchical

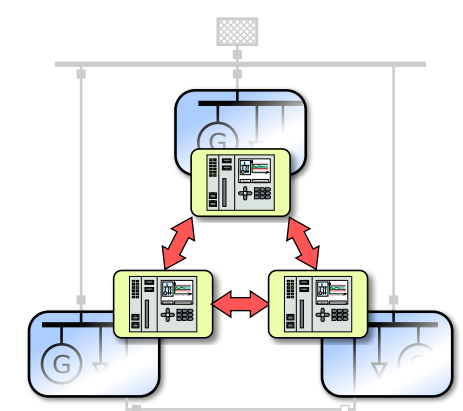

(c) Distributed

Figure 7. Control architectures.

Before the model is solved, it is regularly preprocessed. Nine contributions directly formulate the model as MILP or Mixed Integer Programming (MIP) models (e.g., [20,51,62]) that can be directly solved by commercial solvers. Other models such as $[52,57,60]$ are initially formulated without any restrictions or based on a quadratic formulation $[53,63]$. After stating the model, 
linearization is often applied to reduce the computational burden and to allow the usage of external solvers $[20,54,56,62]$. One contribution also applied an intermediate convexification step before linearizing the problem [56]. One may note that [62] is listed as MILP model and as presenting a linearization, because the power-flow model is stated in its linearized version only, but the linearization procedure is quickly sketched.

Table 5. Optimization approaches ( $\checkmark$ : Implemented, ?: Not reported) .

\begin{tabular}{|c|c|c|c|c|c|c|c|c|c|c|}
\hline Ref. & Method & Location & Formulation & Model Type & 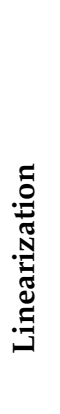 & 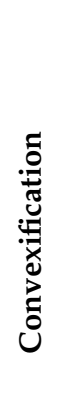 & 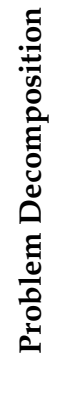 & 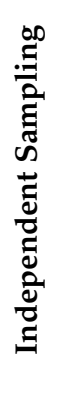 & 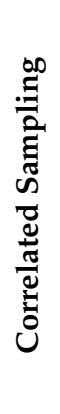 & 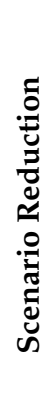 \\
\hline [48] & Mathematical & Central & Stochastic & MIP & & & $\checkmark$ & & $\checkmark$ & $\checkmark$ \\
\hline [49] & ICA & Central & Stochastic & Unconstrained & & & & & & \\
\hline [27] & Mathematical & Central & Deterministic & MIP & & & $\checkmark$ & & & \\
\hline [50] & CSA & Central & Stochastic & Unconstrained & & & & $\checkmark$ & & $\checkmark$ \\
\hline [20] & Mathematical & Central & Stochastic & Unconstrained & $\checkmark$ & & & $?$ & $?$ & $\checkmark$ \\
\hline [51] & Genetic+Mat. & Central & Stochastic & MILP & & & $\checkmark$ & $\checkmark$ & & $\checkmark$ \\
\hline [52] & PSO+Mat. & Central & Stochastic & Unconstrained & & & $\checkmark$ & & & \\
\hline [53] & Mathematical & Central & Deterministic & Quadratic & $\checkmark$ & & $\checkmark$ & & & \\
\hline [28] & Genetic & Central & Stochastic & MIP & & & & & $\checkmark$ & $\checkmark$ \\
\hline [54] & Mathematical & Hierarchical & Deterministic & Unconstrained & $\checkmark$ & & $\checkmark$ & & & \\
\hline [55] & EMA & Central & Stochastic & MIP & & & & & $\checkmark$ & \\
\hline [56] & Mathematical & Central & Stochastic & Unconstrained & $\checkmark$ & $\checkmark$ & $\checkmark$ & & $\checkmark$ & \\
\hline [57] & $\mathrm{PaCcET}$ & Central & Stochastic & Unconstrained & & & & & & \\
\hline [58] & Mathematical & Central & Stochastic & MIP & & & & $?$ & $?$ & \\
\hline [59] & Mathematical & Central & Deterministic & MILP & & & & & & \\
\hline [60] & Mathematical & Central & Deterministic & Unconstrained & $\checkmark$ & & $\checkmark$ & & & \\
\hline [61] & Mathematical & Central & Stochastic & MILP & & & & & $\checkmark$ & \\
\hline [62] & Mathematical & Central & Deterministic & MILP & $\checkmark$ & & $\checkmark$ & & & \\
\hline [63] & Mathematical & Distributed & Stochastic & Quadratic & & & $\checkmark$ & $\checkmark$ & & \\
\hline [64] & PSO & Central & Stochastic & Unconstrained & & & $\checkmark$ & $\checkmark$ & $\checkmark$ & $\checkmark$ \\
\hline
\end{tabular}

One common measure to increase the computational tractability of the scheduling problem [53,62], to develop sub-problem-specific solution procedures [51], and to account for the inherent structure of the scheduling problem $[27,54]$, is to decompose the whole problem into several sub-problems. Eleven out of 20 papers decompose the scheduling such that the partial problems can be solved individually and will then be combined into the complete problem. Since the sub-problems are usually not fully independent, iterative approaches like in $[27,52,54]$ that refine the individual problems are commonly seen.

Robust and stochastic formulations are by far the most common methods to deal with uncertainty in scheduling problems. None of the key contributions considered other means such as fuzzy sets to directly represent uncertainty. In Table 5, the optimization is considered stochastic, in case the optimum is defined with respect to some probabilistic information. In case a robust algorithm computes a single worst case, the optimization procedure itself is categorized as deterministic. Although in some cases, probabilistic models may be optimized without sampling a set of scenarios first [49,52], most papers that follow a stochastic approach report sampling-based methods to generate a certain set of scenarios (e.g., [50,51,64]). These samples are either drawn independently [51], or in an approach which establishes a correlation that is not covered by the sampled distribution and which thereby 
reduces the number of scenarios that need to be considered [55]. To contain the number of samples and to decrease the computational burden, correlated sampling approaches that directly limit the number of samples [61] or scenario reduction techniques that reduce a large amount of scenarios [28] are commonly observed.

On a common ground, all key contributions minimize the operating costs of the controlled microgrids. Except some common terms such as the cost of controllable DERs; however, a wide variety of objective terms is observed and summarized in Table 6. The high prevalence and diversity of cost-based objectives can also be found in other areas of microgrid-related research [10,44]. Despite some broadly used terms including DER operation expenses, some costs such as fees for lost loads are more specific to resilient and islanded microgrid problems. Other terms including investment cost are commonly found in planning problems but do not play a major role in operation. All but four key contributions directly state a single-objective function without considering a multi-objective formulation, first. Three of the papers that consider a multi-objective formulation apply fuzzy decision making to obtain a single schedule automatically $[28,51,57]$. Herein, the decision maker's satisfaction with each single-objective value is encoded as fuzzy membership function and used to select the best compromise solution in the Pareto front. However, uncertainties in the power system are not modeled as fuzzy sets.

In addition to linear (e.g., [27,59,66]) or non-linear (e.g., [63,64]) generation costs of schedulable DERs, start-up and shut-down costs like in [60] are frequently considered. Similar to the DER generation cost, the cost of energy that is sold to or purchased from the main grid (e.g., [28,59]), as well as the value of lost load (e.g., [51,61]) are included by a majority of 19 and 14 contributions, respectively. For instance, [53] considered the value of lost loads via elastic load prices while [51] directly included the value in the unscheduled islanding scenarios. The costs of Vehicle to Grid (V2G) or EES operation is considered by ten key contributions such as [28,50,51,60]. Presented cost terms include cost of the charged and discharged energy [53], constant start-up, shut-down and operation fees [60], as well as detailed EV battery degradation models [50].

In contrast to regularly considered tariffs of transferring energy from or to an external main grid, trading energy between independent microgrids is considered by a minority of five key contributions including $[27,58]$. Other rarely used cost terms include maintenance costs [28], the cost of power losses [55], the cost of scheduling additional load, for instance in DR programs [20,61], costs or benefits gained by selling energy to customers [28,53], costs for committing operational reserves without actually scheduling them [52], and cost terms that penalize uncertainty or security margins $[57,62]$. Two out of the four contributions that considered remote switches also considered the cost of operating them [50,55]. Although one key contribution [52] directly considered the emission of pollutants, no publication added emission costs such as the cost of $\mathrm{CO}_{2}$ emissions. 
Table 6. Objective terms ( $\checkmark$ : Implemented).

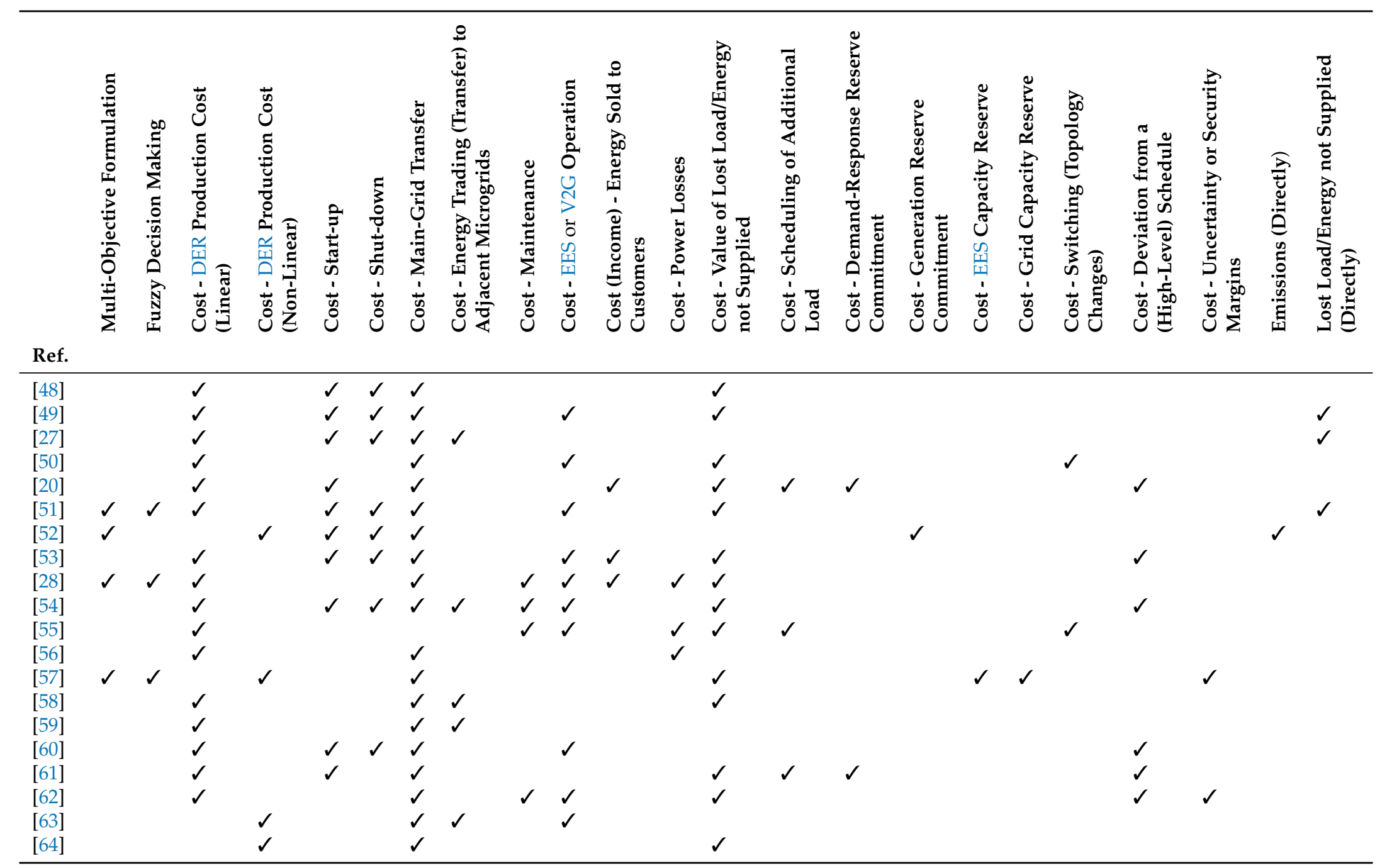




\subsection{Resilience Features}

Although a microgrid and multi-microgrid may be impacted by a wide variety of failures [14] such as communication faults, misbehaving circuit breakers, and degraded batteries, only a small subset of these failure modes is commonly considered. Table 7 summarizes the observed failure modes and mitigation techniques. A great majority of 17 key contributions including $[48,58,61]$, incorporated main-grid outages and nine publications such as [51,60] considered this mode as the only source of unplanned disturbances. Eight contributions hardened their scheduling approaches by considering line tripping events (e.g., $[48,54,74]$ ) and three publications assumed that a generator may fail $[48,55,62]$.

Notably, [50] generically considered bus faults or disconnections and corresponding fault isolation and rerouting actions. Generic deviations from the scheduling decision are addressed by [63] in which an adversary model that includes intentional attacks as well as faults of the scheduled assets is formulated. A detailed, transient short-circuit model is used by one key contribution, [57], that assessed each candidate schedule by a set of transient simulations. Following the eligibility criteria, all failures are either considered to be stochastic phenomena given a stochastic failure model [61] or as indeterministic phenomena that could either happen all the time or in exceptionally vulnerable situations [27,53].

Depending on the currently active schedule, a multi-microgrid may have several options to mitigate the effects of a failure. All mitigation techniques that were found in the key contributions are illustrated in Figure 8. When an external fault happens, most of the scheduling approaches consider the disconnection from the main grid as the primary mitigation measure. In particular, eighteen contributions such as $[52,62,64]$ include islanding options in their schedule. Ten of these publications including $[27,49,59]$, solely focus on islanding and do not actively consider other fault mitigation techniques in their scheduling approaches.

Other fault mitigation options are also found in the literature. Grid splitting, for instance, which refers to the division into several independent sections which are not electrically connected, is considered by seven key contributions (e.g., [55,61,62]). In addition, a failure may be compensated by establishing another connection to islanded or disconnected parts of the network $[48,54,63]$. Rerouting is considered by a minority of six publications as an alternative method of compensating failures.

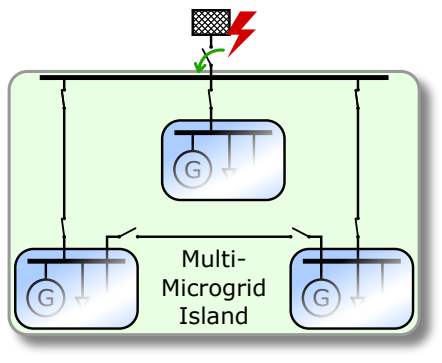

(a) Islanding

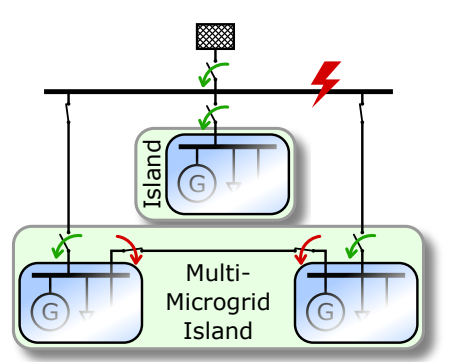

(b) Grid splitting

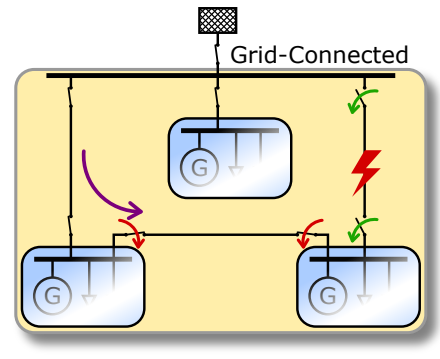

(c) Rerouting

Figure 8. Observed mitigation schemes. 
Table 7. Resilience features ( $\checkmark$ : Implemented, ?: Not reported, St: Stochastic, D: Deterministic, ID: Indeterministic) .

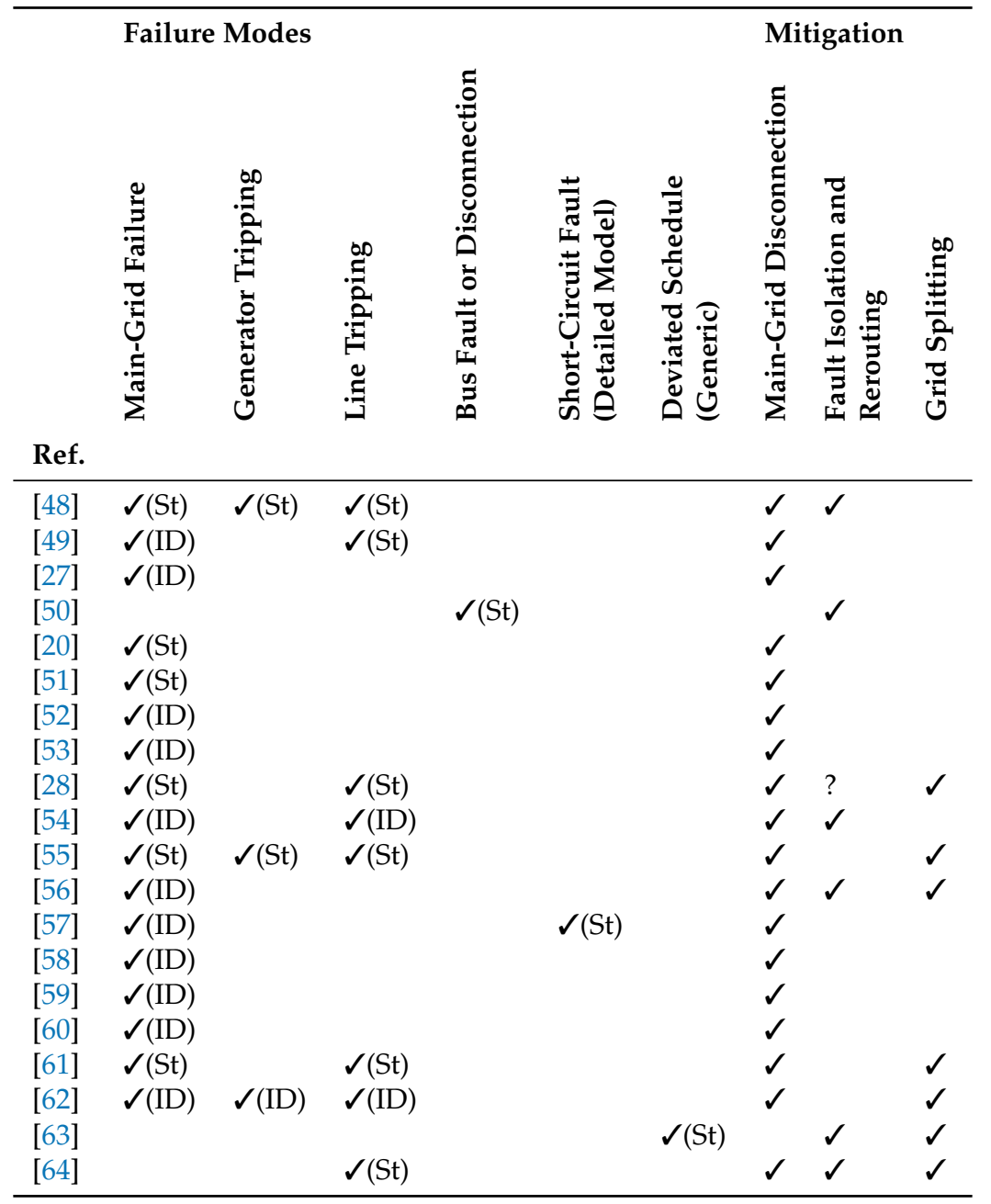

\subsection{Validation Approaches}

Another aspect in the construction of a resilient scheduling approach is its validation. Table 8 summarizes the validation approaches of each key contribution. One can see that all key contributions are validated by numeric simulations. However, only one single key contribution, [59], also includes a laboratory test setup and none of these publications reported any field tests or real-world deployments. Most of the publications assessed the performance in one or multiple failure conditions, either explicitly based on single scenarios $[27,57,62]$ or via some performance metric that aggregate multiple failure conditions [20,51]. Following the modes summarized in Table 7, mostly main-grid failures such as in [27] are assessed. However, other failures such as tripping generators [62], line failures [28], deviated schedules [63], deviations from forecasts or from input parameters [59], as well as short-circuit events [57] were also included. 
Table 8. Validation approaches ( $\checkmark$ : Implemented, ?: Not reported)

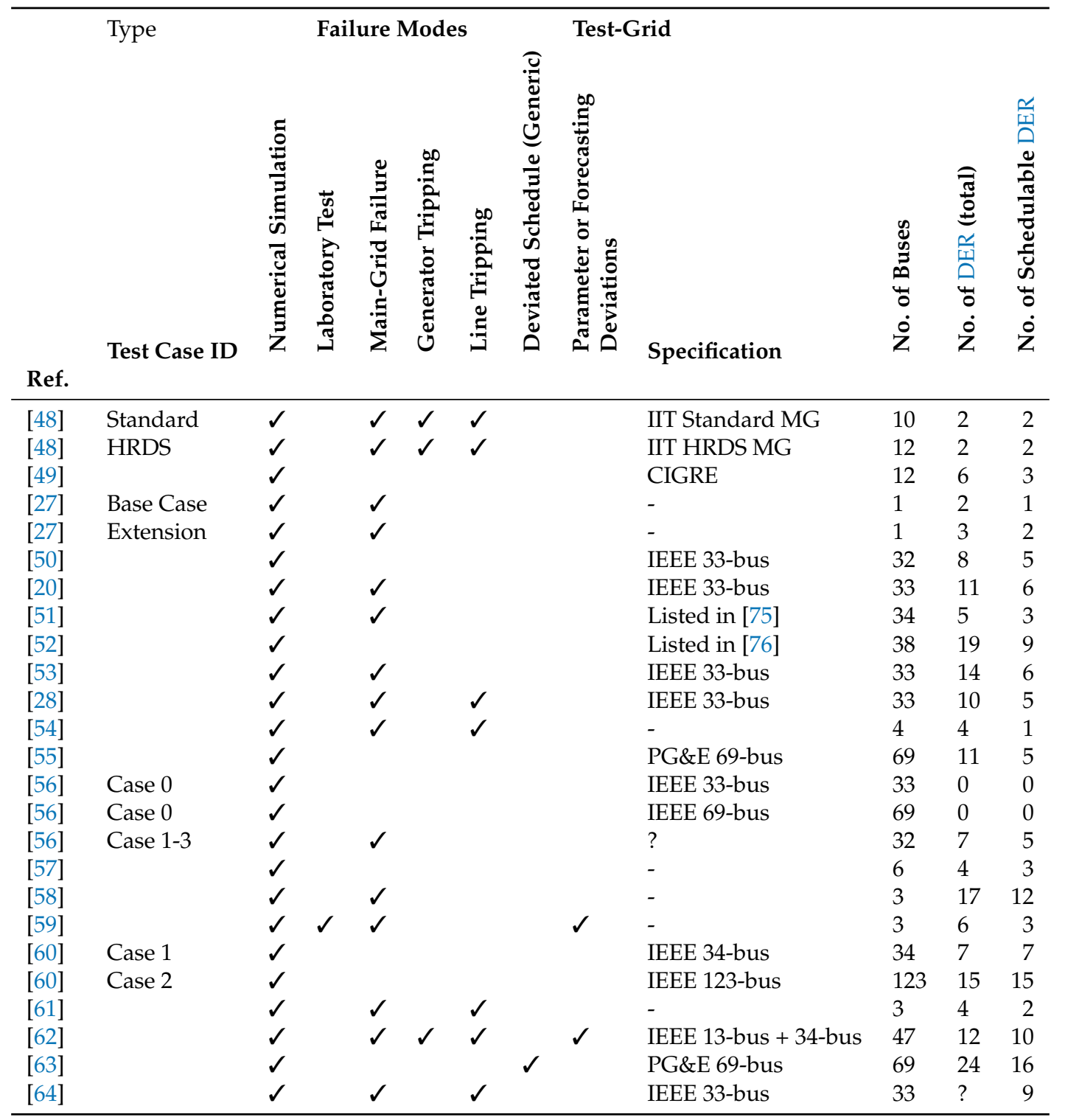

In most cases, one single test grid is used to assess the scheduling methodology. Exceptions include [48], which uses multiple variations of a single network and [60] which uses two different test networks. Six times, including [28,50,53], the assessment was based on a grid called Baran or IEEE 33-bus test feeder [77]. Other test systems include the official IEEE 34-bus, and 123-bus test feeders [60], as well as some CIGRE benchmark systems [49]. To be able to test assets that were not considered in the initial test network, one commonly observed practice is the extension by various DERs $[53,60]$. The sizes of assessed networks range from one bus systems that host up to three DERs [27] to 123-bus grids [60] and networks that host up to 24 DERs [63].

Typically, the scheduling algorithm is executed once per day and the next schedule is calculated. In particular, 16 key contributions such as [49,54,59], configure a scheduling horizon of $24 \mathrm{~h}$. For [48], the schedule of a whole year is assessed, but no other information on the scheduling horizon was given. The observed time resolution ranges from minutely scheduling intervals [59] to hourly set-points like in $[52,57]$ that are used in 14 key contributions.

A minority of two publications implements a scheduling scheme that updates or installs set-points before the end of one scheduling horizon. A scheduling function that updates parts of the optimization both in real time and as soon as new forecasts are available, is presented by [60]. A fully cyclic 
approach that outputs the first set-point of each scheduling horizon only, is presented in [63]. Figure 9 illustrates the observed scheduling schemes and shows an exemplary scheduling output for three controllable assets. Table 9 summarizes the main features of the validation platforms and the execution parameters. One can see that only few publications give hints as to the computational complexity of their algorithms by presenting the execution time [20,54,60,61]. However, the software tools such as MILP solvers and hardware platforms are listed more commonly.

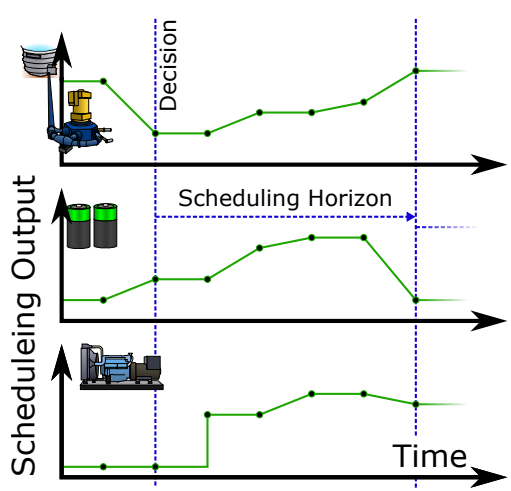

(a) Run Once

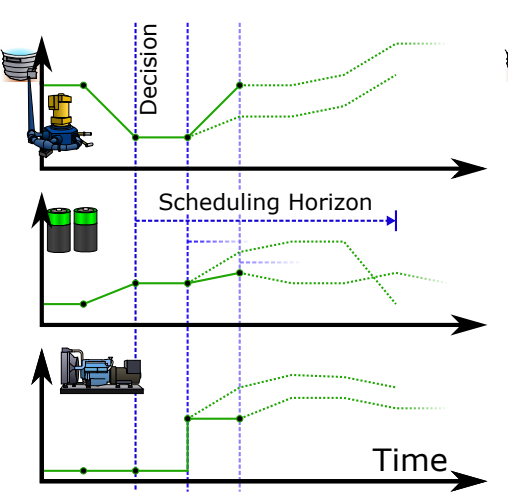

(b) Fully Cyclic

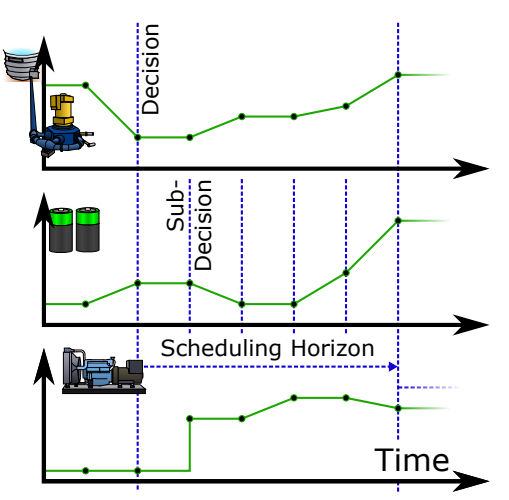

(c) Interleaved

Figure 9. Observed scheduling schemes.

To validate a schedule, several input profiles such as load and RES generation are needed. Table 10 summarizes type and source of load, energy price, PV and wind generation, as well as EV charging profiles. For wind and PV profiles, both electricity generation $[51,66]$ and primary energy source profiles [28] are subsumed. For [27,52], generation of volatile RES is considered in a generic way, either as negative loads or as generic generation. In the first case, only load profiles are listed in Table 10, while in the second case, the RES profile is listed for both PV and wind generation.

Since most related models are stochastic or indeterministic in nature, profiles are often not only given as single deterministic curves, but as distribution or set of feasible values. Most papers such as $[53,57,64]$ base their undetermined inputs on a deterministic forecast and model the forecasting errors in addition. In terms of forecast errors, both stochastic formulations including $[50,58]$ and indeterministic ones such as $[51,66]$ are regularly observed. One can see that most of the publications that use a stochastic model assume that the observables or forecasting errors are temporally independent, i.e., one sample does not depend on any previous sample.

A rich set of evaluation metrics, as summarized in Table 11, is used to assess the performance of scheduling algorithms. The most common terms are operation costs or their stochastic expectation (e.g., [56,57]) that is used by 15 key contributions. Eleven publications such as $[28,60]$ published results regarding energy transfer either between different microgrids or to the main grid. Half of the key contributions including [55,62] provided information on bus voltages and seven listed the expected energy that cannot be supplied (e.g., [51,58]). In addition to the frequent metrics, several metrics such as network losses [51], the system average interruption duration index [48], and pollutant emissions [52] were given. 
Table 9. Validation platform (?: Not reported).

\begin{tabular}{|c|c|c|c|c|c|c|}
\hline Ref. & Test Case ID & 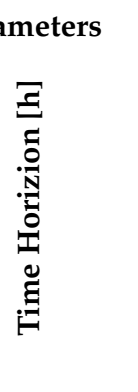 & 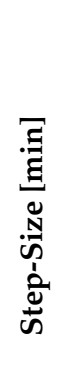 & 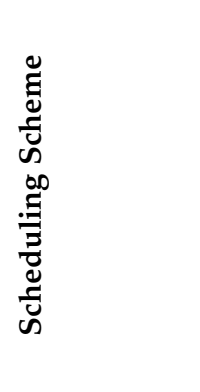 & 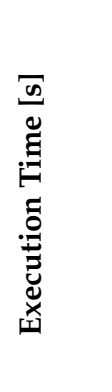 & Reported Execution Platform \\
\hline [48] & Standard & $8760(?)$ & 60 & Run-Once & & \\
\hline [48] & HRDS & $8760(?)$ & 60 & Run-Once & & \\
\hline [49] & & 24 & & Run-Once & & MATLAB implementation \\
\hline [27] & Base Case & 24 & 60 & Run-Once & & CPLEX 11.0, 2.4GHz PC \\
\hline [27] & Extension & 24 & 60 & Run-Once & & CPLEX 11.0, 2.4GHz PC \\
\hline [50] & & 24 & 60 & Run-Once & & \\
\hline [20] & & 24 & 60 & Run-Once & 22.59 & Intel Core i7 CPU, 3.20 GHz, 4GB RAM, IBM ILOG CPLEX 12.4, GAMS IDE \\
\hline [51] & & 24 & 60 & Run-Once & & MATPOWER \\
\hline [52] & & 24 & 60 & Run-Once & & MATPOWER (MINOPF) \\
\hline [53] & & 24 & 60 & Run-Once & & Intel Core i7 CPU, $3.20 \mathrm{GHz}$, 4GB RAM, IBM ILOG CPLEX 12.4, GAMS IDE \\
\hline [28] & & 24 & 60 & Run-Once & & MINLP model, NSGAII algorithm, MATLAB 2016a \\
\hline$[54]$ & & 24 & 15 & Run-Once & 124.37 & MATLAB, YALMIP toolbox, CPLEX 12.4, 32-bit PC, 2.10GHz CPU, 2GB RAM \\
\hline [55] & & 24 & 60 & Run-Once & & \\
\hline [56] & Case 0 & - & - & Run-Once & & CPLEX 11.0, 2.4-GHz PC \\
\hline [56] & Case 1-3 & 24 & 60 & Run-Once & & CPLEX 11.0, 2.4-GHz PC \\
\hline [57] & & 5 & 60 & Run-Once & & Win7, E5420 CPU, 2 Cores, $2.5 \mathrm{GHz}, 16$ GB RAM \\
\hline [58] & & 24 & 60 & Run-Once & & GAMS, BARON \\
\hline$[59]$ & & 24 & 1 & Run-Once & & IBM CPLEX \\
\hline [60] & Case 1 & 24 & 30 & Interleaved & 9.8 & OpenDSS + OPTI Toolbox + MATLAB, Windows, Intel Core i5-4300M, 2.60GHz, 8 GB RAM \\
\hline [60] & Case 2 & 24 & 30 & Interleaved & 36.9 & OpenDSS + OPTI Toolbox + MATLAB, Windows, Intel Core i5-4300M, 2.60GHz, 8 GB RAM \\
\hline [61] & & 24 & 60 & Run-Once & 0.06 & GAMS DIE, CPLEX 12.4, Intel Core i7 CPU, 3GHz, 12 GB RAM \\
\hline [62] & & 6 & 15 & Run-Once & & Python 2.7, GUROBI 8.0.0, Intel Core i7-7700, 4.2 GHz, 16GB RAM \\
\hline [63] & & 1 & 15 & Fully Cyclic & & MATLAB with YALMIP, Intel Core i7, $2.6 \mathrm{GHz}, 16 \mathrm{~GB}$ RAM \\
\hline [64] & & 24 & 60 & Run-Once & & \\
\hline
\end{tabular}


Table 10. Input profiles (Det.: Deterministic, Dist.: Distribution, Int.: Interval, Dev.: Deviation, TI: Temporally Independent, ID: Indeterministic, N.A.: Not Applicable, UnAv:: Source is Unavailable, -: Source is not listed).

\begin{tabular}{|c|c|c|c|c|c|c|c|c|c|c|}
\hline \multirow[b]{2}{*}{ Ref. } & \multirow{2}{*}{$\begin{array}{l}\text { Load } \\
\text { Type }\end{array}$} & \multirow[b]{2}{*}{ Source } & \multicolumn{2}{|c|}{ Main-Grid Energy Prices } & \multirow{2}{*}{$\begin{array}{l}\text { PV/Irradiation } \\
\text { Type }\end{array}$} & \multirow[b]{2}{*}{ Source } & \multirow{2}{*}{$\begin{array}{l}\text { Wind } \\
\text { Type }\end{array}$} & \multirow[b]{2}{*}{ Source } & \multicolumn{2}{|l|}{ EV Charging } \\
\hline & & & Type & Source & & & & & Type & Source \\
\hline [48] & Det. & - & Det. & UnAv. & N.A. & - & N.A. & - & N.A. & - \\
\hline [49] & Det. & - & Det. & UnAv. & Det. & - & Det. & - & N.A. & - \\
\hline [27] & Det. + TI Dist. & - & Det. + TI Dist. & - & N.A. & - & N.A. & - & N.A. & - \\
\hline [50] & Det. + TI Dist. & - & Det. + TI Dist. & - & Det. + TI Dist. & - & Det. + TI Dist. & - & Det. + TI Dist. & - \\
\hline [20] & Dist. & [78] & Dist. & [78] & N.A. & - & TI Dist. & [79] & TI Dist. & - \\
\hline [51] & Det. + TI Dist. & [80] & Det. & - & Det. + TI Dist. & [80] & Det. + TI Dist. & [80] & N.A. & - \\
\hline [52] & Det. + TI Dist. & - & Det. & [81] & Det. + TI Dist. & - & Det. + TI Dist. & - & N.A. & - \\
\hline [53] & Det. + Det. Dev. & [20] & Det. + ID Int. & [20] & N.A. & - & N.A. & - & N.A. & - \\
\hline [28] & Det. + TI Dist. & - & Constant & - & TI Dist. & - & TI Dist. & - & N.A. & - \\
\hline [54] & Det. + ID Int. & - & Det. & - & Det. + ID Int. & - & Det. + ID Int. & - & N.A. & - \\
\hline [55] & Det. & - & Det. & - & Dist. & - & Dist. & - & N.A. & - \\
\hline [56] & Det. + Dist. & - & Det. + Dist. & - & N.A. & - & Det. + Dist. & - & N.A. & - \\
\hline [57] & Det. & - & Det. & - & Det. & - & N.A. & - & N.A. & - \\
\hline [58] & Det. + TI Dist. & - & Det. & - & Det. + TI Dist. & - & Det. + TI Dist. & - & N.A. & - \\
\hline [59] & Det. + Det. Dev. & [82] & Det. & - & Det. + Det. Dev. & [83] & N.A. & - & N.A. & - \\
\hline [60] & Det. & - & Det. & - & Det. & - & Det. & - & N.A. & - \\
\hline [61] & Dist. & [84] & Dist. & [84] & N.A. & - & Dist. & [84] & N.A. & - \\
\hline [62] & Det. + ID Int. & [85] & Det. & [86] & N.A. & - & Det. + ID Int. & [87] & Constant & [88] \\
\hline [63] & Det. + TI Dist. & UnAv. & Constant & - & Det. + TI Dist. & UnAv. & N.A. & - & N.A. & - \\
\hline [64] & Det. + TI Dist. & - & Det. + TI Dist. & - & Det. + TI Dist. & - & Det. + TI Dist. & - & N.A. & - \\
\hline
\end{tabular}


Table 11. Evaluation metrics ( $\checkmark$ : Implemented).

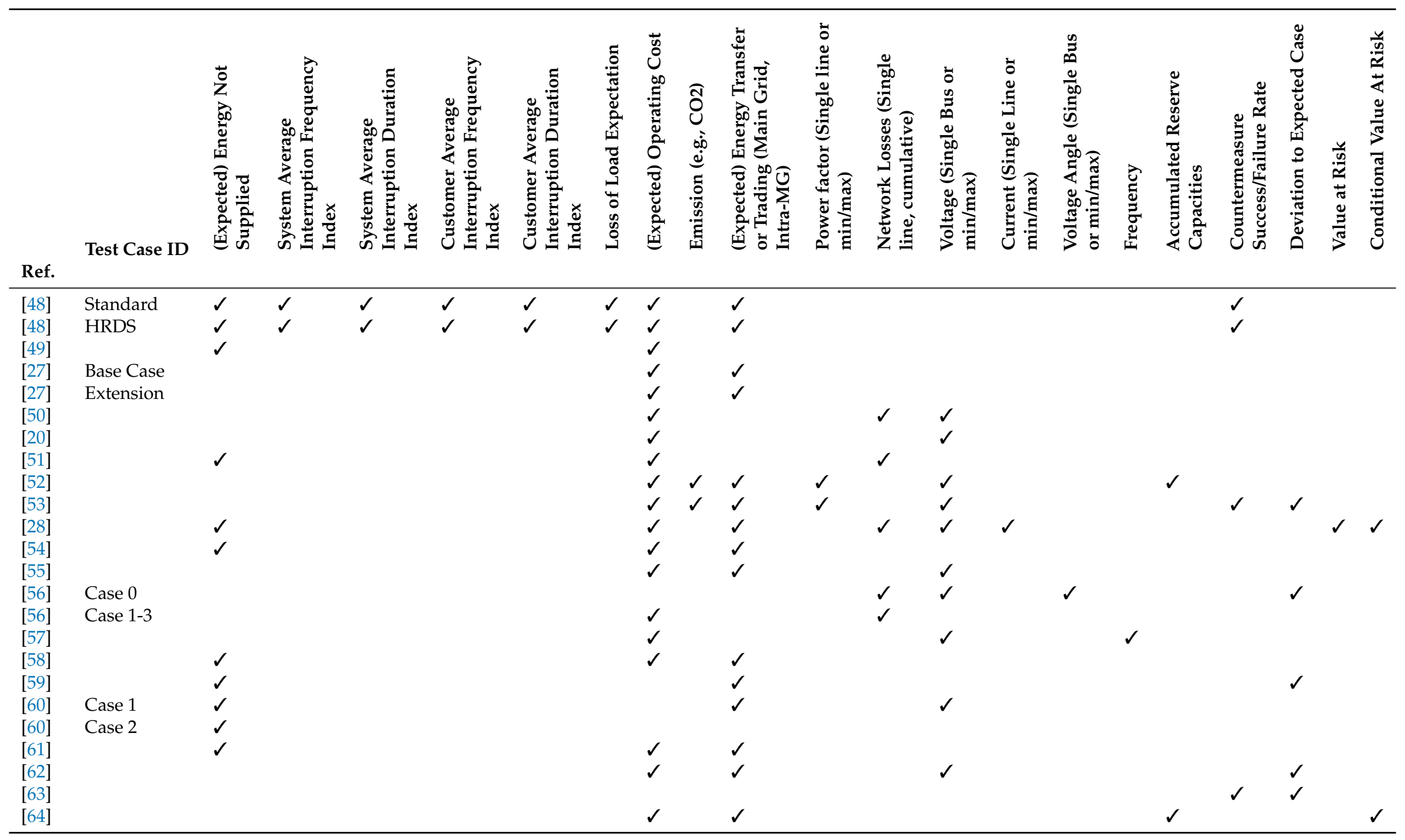




\section{Future Perspectives}

Several key publications, as summarized in Table 2, demonstrated the feasibility of resilient scheduling in multi-microgrids or provided significant contributions that may also be applied in such an environment. Several design choices were successfully explored, and a broad spectrum of scheduling approaches was presented. However, none of the key contributions reported a field implementation. Additionally, practical aspects such as the availability and quality of input data are rarely discussed. To guide future research towards practical implementation and alternative options that may be applied as well, a list of open points is given.

\subsection{Modeling Approaches and Modeled Assets}

Although one can observe a broad variety of asset models, there are various research opportunities to refine knowledge of the effects of modeling assumptions. These opportunities include the following perspectives on modeling approaches and asset models.

- Level of Abstraction: Most assets such as loads, generation and grid facilities were modeled by different levels of abstraction. For instance, some papers consider a graph model and active power-flow constraints, only [54], while others include a detailed grid model [20] that considers reactive and apparent power flows, as well. Choosing the right level of abstraction may drastically influence the system performance [56], but few papers present the impact of simplification on a resilient schedule in detail.

- Hidden Aspects: To get a concise view on the required level of abstractions, several aspects, that are usually not modeled but that may impact an algorithm, should be considered. For instance, storage losses are commonly modeled via constant efficiencies. However, battery storage systems including their power electronics converters show various non-linear effects that strongly depend on the point of operation [89,90]. A more detailed model may cover system effects that potentially hamper supply security in critical part-load situations [3]. Similarly, few papers consider the effects and reserve requirements of low-level controls such as $Q$-of- $U$ and $P$-of- $f$ droop curves that may implement short-term power sharing and voltage control [17].

- Stochastic Effects: Following one of the eligibility criteria, all key contributions trivially consider stochastic effects such as volatile load and RESs. Some of these contributions directly use stochastic models of meteorological observables and forecasting deviations. On a common basis, errors and the observables themselves are assumed to be independently distributed as concerns points in time, i.e., one sample does not depend on previous samples. Such assumptions may be valid for yearly assessments, but may fail at short-term horizons [70] of scheduling problems. Work [91] studies forecasting errors and indicates that a commonly assumed normal distribution does not fit either. Some research is needed to quantify the effects on the performance of a resilient scheduling algorithm and to study alternative models.

- Robustness of the Asset Models: Asset models may be subject to parameter deviations and degradation. For RESs and loads, such phenomena are commonly considered, but for controlled assets, the effects of parameter deviations such as EES capacities are hardly covered. Future work may tackle the robustness of asset models by studying the effects of inevitable parameter deviations. A feedback mechanism may be included to assure a sufficient model quality [92] and further insights regarding the impact of inaccuracies may be gained.

- Covered Assets: Current scheduling algorithms focus on a specific set of assets such as generic DER, EES, and constant power loads. Other components such as transformers that are equipped with On-Load Tap Changers (OLTCs) are not included in the key contributions. However, these assets may impact the system performance and may even be actively controlled by the scheduling algorithms, e.g., to support voltage control via OLTCs [93]. In case specific plant types such as hydro turbines and hydrogen-based EES may be subsumed by generic models, the eligibility of these models needs to be assessed as well. 
- Engineering Aspects: One open point that is beyond the scope of all key contributions is the efficient engineering of scheduling approaches. All approaches require a large amount of input information such as network and DER parameters that may not be easily available. Methods that inherently include a system and model identification process such as reinforcement learning [94], as well as common data sources and engineering support systems [95] may be used to assist practical implementation.

\subsection{Optimization Objectives and Methods}

Optimization procedures and objectives are an integral part of each key contribution. Due to that relevance, significant impact may be gained by refining optimization-related aspects and by applying findings from related fields.

- Distributed Optimization: Out of 20 contributions, only a single one followed a fully distributed approach [63]. Most other publications implemented a centralized approach that requires extensive knowledge of the assets of various microgrids. Future work may include resilience aspects in distributed scheduling approaches more often [34] or may distribute centralized algorithms to tackle privacy, fault tolerance, and governance issues [35].

- Hybrid Optimization: Traditionally, optimization approaches either use mathematical programming or heuristics to obtain a feasible and possibly optimal solution [73]. However, there is a class of algorithms called hybrid optimization that tries to combine several complementary methods, for instance both mathematical programming and heuristic procedures. Some key contributions already successfully use hybrid approaches, e.g., for problem decomposition $[27,53]$ or to interface external solvers [51,57]. Future work may improve the interaction between different solvers and study the application of hybrid optimization methods in detail. A particular focus may be put on the integration of detailed models that are solved by external tools.

- Scalability: Some papers such as [53] indicate that techniques to improve the performance are needed, but as listed in Table 9, few publications actually assess the execution time. Furthermore, no systematic scalability study is given that relates the execution time to the system size and complexity. It is still open to assess the scalability of scheduling approaches and to document the performance limits with respect to practical systems. A fine-grained evaluation may highlight performance trade-offs and assist future engineering work.

- Regulatory Frameworks: Although the key contributions include a wide variety of cost terms, including resilience and operating costs, few references to regulatory regimes that imply certain cost structures are given. Such frameworks may dictate when price information is available, how prices are determined and whether a market can be accessed [96]. Furthermore, energy transfer and trading within multi-microgrids or local energy communities may be restricted by various regulations that need to be considered in the design and operating phase. Some key contributions already consider regulatory measures by assuming certain market price structures [20,62], but a broad discussion is missing.

\subsection{Resilience Features}

One major aspect of this review is the resilience of scheduling algorithms that manage normal operation. As such, all key contributions already consider some failure modes and fault mitigation techniques. However, the analysis revealed several research opportunities that may increase the resilience and robustness of microgrids even further.

- Extended Failure Modes: In most key contributions, only a few failure classes that must be withstood are considered. Typically, main-grid faults $[27,52]$ and line tripping events $[28,64]$ are tackled. Few papers also include other modes such as short-circuit failures [57] and no paper directly considers faulty DER set-points, invalid switching actions, or load disconnections. Open research includes the systematic identification of relevant failure modes that need to be considered in the 
scheduling problem. Additionally, the flexibility of algorithms in considering new and previously unknown failures may be assessed. Future work may further rely on the integration of external simulation tools to tackle both flexibility and the level of detail.

- Generic Failure Modes: Some failure modes may be subsumed by another class. For instance, main grid failures may be covered by a fault in the line that connects the point of common coupling $[28,55]$. However, a systematic study of prototypical failure modes in scheduling problems is still open. In particular when increasing the number of considered failure modes, a proper generalization may be needed to contain the computational effort of finding an optimal solution.

- Effects of Low-Level Controls: Low-level controls such as voltage and frequency control that are installed to provide immediate action in terms of disturbances [5] are commonly beyond the scope of key contributions. However, a high-level schedule may impact the feasibility of certain control actions, e.g., in case a generator is already close to a limit, and may hamper a successful mitigation action. Some emergency actions such as fault rerouting can also be implemented by a dedicated controller to guarantee a fast response. Future work may focus on the interaction of low-level controls and the scheduling algorithm to ensure a consistent behavior and a valid emergency response. Insights into the proper abstractions of low-level controls may decrease the complexity of the scheduler without impacting the resilience of the system.

- Fault Mitigation Techniques: The key contributions deploy three different classes of fault mitigation techniques: Main-grid disconnection [27], rerouting [48], and grid splitting [28]. However, practical issues such as protection, inertia, grid-forming, and legal requirements [36] are hardly considered on assessing mitigation options. Some work may be conducted to increase the knowledge of feasibility aspects of mitigation techniques and to explore further options such as mobile generators and batteries that may quickly replace other units [97].

\subsection{Validation Approaches}

The key contributions mostly focus on simulation-based validation, only. Considerable work is needed until the reviewed algorithms can be safely and efficiently applied in real-world setups. The following research opportunities target the progression of technological readiness.

- Common Benchmark System: All key contributions include a simulation-based validation, but each of them uses different benchmark systems or diverging configurations to demonstrate their approaches. One of the most common test feeders is the Baran test feeder [77]. However, to demonstrate the algorithms, several independent modifications were introduced to account for DERs that were not considered in the original test feeder. Consequently, results from different papers cannot be directly compared. Existing benchmark systems [31] such as SimBench [98,99] and IEEE test feeders [100] may be evaluated and refined towards a unified multi-microgrid scheduling test bed. In addition to common typologies, a wide variety of assets, unified input profiles, and detailed DER parameters may reduce the need for custom modifications.

- Common Metrics: To be able to compare results from different studies, common metrics are required. While most papers provide operating costs, resilience-based metrics such as energy not supplied, are less common. Future work may profit from an increased focus on unified resilience metrics [48] and may intensify the discussion on their significance.

- Independent Validation: Rising from the need for comparable results, a fine-grained assessment of multiple algorithms may be conducted. In addition to common benchmark systems that are used by multiple authors, more detailed insights may be gained. A common execution platform may, for instance, enable detailed assessments on the computational performance. Some publications including $[56,64]$ already compare their methods to a reference, but future implementations may profit from a more extensive study. 
- Readiness and Practical Implementation: Although the first multi-microgrid test sites are already implemented [9], only one key contribution demonstrated the practical implementation of resilient scheduling [59] and no one went beyond laboratory experiments. Hence, considerable effort is needed until the approaches can demonstrate practical use in real-world situations [101]. Future testing and validation work may highly profit from previous experiences $[9,31,102]$ and structured testing methodologies such as holistic testing [101,103].

- Resilience Assessment in the Field: One particular challenge towards a safe deployment is the resilience assessment in productive operation. Unlike in laboratory-based setups, testing the resilience may impact the overall performance of the grid. However, undiscovered failures in the control strategies may have fatal consequences as well [30]. Future work may include save strategies to verify resilience against low probability, high impact events in operation.

\section{Conclusions}

Resilient, proactive scheduling in the context of multi-microgrids is currently at a development stage that bears a manifold variety of optimization-based approaches but lacks practical experiences in implementing these methods beyond simulation-based test beds. However, practical experiences in related fields such as first insights into the operation of multi-microgrids in general, show a considerable potential in enhancing both security and cost of conventional electricity supply. The study systematically identifies the main contributions in proactive, resilient multi-microgrid scheduling and provides an in-depth analysis of selected literature. The typical scheduling approach uses an optimization-based framework that minimizes the running costs while meeting several resilience and operation constraints. However, the wide variety in modeling, solving, and validation of these scheduling problems raises the need for the presented detailed discussion. Several design decisions and the current spectrum of approaches are identified to aid future refinements and to back first practical implementations alike.

Corresponding to the current stage of development, several open issues and future perspectives were identified. Considerable work needs to be done in validating existing approaches and assessing the performance in practical implementations. Research work that compares various scheduling algorithms on a common ground is needed to establish a common view on the scheduling performance and to guide towards field tests. Similarly, engineering aspects should be emphasized to ease a future, widespread application. Also, a considerable potential of methodological improvements was identified. For instance, the quality of asset models may be raised by studying the robustness, limits, and practical applicability of common modeling assumptions. Solution approaches such as distributed optimization that show benefits in related fields may be increasingly applied for resilient multi-microgrid scheduling as well. Other potential improvements include systematic studies on extended failure models and novel fault mitigation measures that may strengthen the resilience of the scheduling outcome even further. Finally, a broad discussion on opportune applications of multi-microgrid scheduling in comparison to various other approaches such as purely economic scheduling in web of cells, needs to be held. This paper contributes to the discussion on finding the sweet spot of multi-microgrid systems by highlighting the SotA in resilient proactive scheduling.

Supplementary Materials: The following are available at http:/ /www.mdpi.com/1996-1073/13/17/4543/s1. Several search terms were used to identify the key contributions. The list contains the search terms, the date of the search operation, as well as the resulting matches. For each search operation, Google Scholar was used as a search engine. Since subsequent entries were found to be less relevant, the number of considered matches was limited to the first 80 publications. The first three search terms were repeatedly applied to update the list of publications and to identify temporal changes in the search results. To avoid missing out relevant publications, all terms were selected such that a broad spectrum of publications is covered.

Author Contributions: Conceptualization, M.H.S., E.M.S.P.V., and T.I.S.; methodology, M.H.S. and E.M.S.P.V.; validation, M.H.S.; formal analysis, M.H.S.; investigation, M.H.S.; resources, T.I.S.; data curation, M.H.S.; writing-original draft preparation, M.H.S.; writing-review and editing, E.M.S.P.V. and T.I.S.; visualization, M.H.S.; supervision, E.M.S.P.V. and T.I.S.; project administration, T.I.S. All authors have read and agreed to the published version of the manuscript. 
Funding: This research received no external funding

Conflicts of Interest: The authors declare no conflict of interest.

\section{Abbreviations}

The following abbreviations are used in this manuscript:

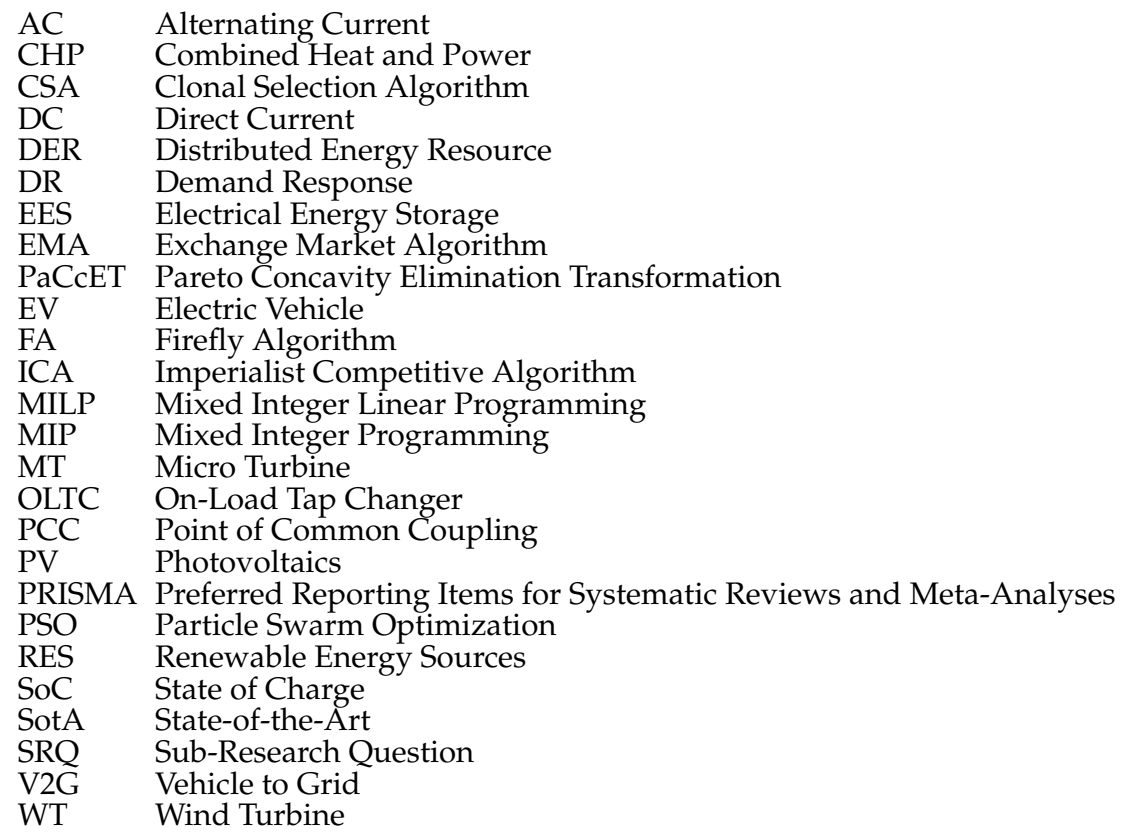

\section{References}

1. Marnay, C.; Abbey, C.; Joos, G.; Ash, K.; Bando, S.; Braun, M.; Chatzivasileiadis, S.; Driesen, J.; Hatziargyriou, N.; Iravani, R.; et al. Microgrids 1 Engineering, Economics, E Experience-Capabilities, Benefits, Business Opportunities, and Examples; Technical report; WG C6.22; CIGRÉ: Paris, France, 2015.

2. Parhizi, S.; Lotfi, H.; Khodaei, A.; Bahramirad, S. State of the Art in Research on Microgrids: A Review. IEEE Access 2015, 3, 890-925. [CrossRef]

3. Hussain, A.; Bui, V.H.; Kim, H.M. Microgrids as a resilience resource and strategies used by microgrids for enhancing resilience. Appl. Energy 2019, 240, 56-72. [CrossRef]

4. Gholami, A.; Aminifar, F.; Shahidehpour, M. Front Lines Against the Darkness: Enhancing the Resilience of the Electricity Grid Through Microgrid Facilities. IEEE Electrif. Mag. 2016, 4, 18-24. [CrossRef]

5. Hatziargyriou, N. (Ed.) Microgrids: Architectures and Control; John Wiley \& Sons: West Sussex, UK, 2014.

6. Kariniotakis, G.; Martini, L.; Caerts, C.; Brunner, H.; Retiere, N. Challenges, innovative architectures and control strategies for future networks: The Web-of-Cells, fractal grids and other concepts. In Proceedings of the 24th International Conference \& Exhibition on Electricity Distribution (CIRED), Glasgow, UK, 12-15 June 2017.

7. Bullich-Massagué, E.; Díaz-González, F.; Aragüés-Peñalba, M.; Girbau-Llistuella, F.; Olivella-Rosell, P.; Sumper, A. Microgrid clustering architectures. Appl. Energy 2018, 212, 340-361. [CrossRef]

8. Shirzadi, S.; Nair, N.K.C. Power system resilience through microgrids: A comprehensive review. In Proceedings of the 2018 IEEE PES Asia-Pacific Power and Energy Engineering Conference (APPEEC), Kota Kinabalu, Malaysia, 7-10 October 2018.

9. Xu, Z.; Yang, P.; Zheng, C.; Zhang, Y.; Peng, J.; Zeng, Z. Analysis on the organization and Development of multi-microgrids. Renew. Sustain. Energy Rev. 2018, 81, 2204-2216. [CrossRef]

10. Khan, A.A.; Naeem, M.; Iqbal, M.; Qaisar, S.; Anpalagan, A. A compendium of optimization objectives, constraints, tools and algorithms for energy management in microgrids. Renew. Sustain. Energy Rev. 2016, 58, 1664-1683. [CrossRef]

11. Arghandeh, R.; von Meier, A.; Mehrmanesh, L.; Mili, L. On the definition of cyber-physical resilience in power systems. Renew. Sustain. Energy Rev. 2016, 58, 1060-1069. [CrossRef] 
12. Gholami, A.; Shekari, T.; Amirioun, M.H.; Aminifar, F.; Amini, M.H.; Sargolzaei, A. Toward a consensus on the definition and taxonomy of power system resilience. IEEE Access 2018, 6, 32035-32053. [CrossRef]

13. Henry, D.; Ramirez-Marquez, J.E. Generic metrics and quantitative approaches for system resilience as a function of time. Reliab. Eng. Syst. Saf. 2012, 99, 114-122. [CrossRef]

14. Li, Z.; Yuan, Y.; Li, F. Evaluating the reliability of islanded microgrid in an emergency mode. In Proceedings of the 2010 45th International Universities Power Engineering Conference (UPEC), Wales, UK, 31 August-3 September 2010 .

15. Falahati, B.; Kargarian, A.; Fu, Y. Timeframe capacity factor reliability model for isolated microgrids with renewable energy resources. In Proceedings of the 2012 IEEE Power and Energy Society General Meeting, San Diego, CA, USA, 22-26 July 2012.

16. Bie, Z.; Zhang, P.; Li, G.; Hua, B.; Meehan, M.; Wang, X. Reliability evaluation of active distribution systems including microgrids. IEEE Trans. Power Syst. 2012, 27, 2342-2350. [CrossRef]

17. Ahn, S.J.; Nam, S.R.; Choi, J.H.; Moon, S.I. Power scheduling of distributed generators for economic and stable operation of a microgrid. IEEE Trans. Smart Grid 2013, 4, 398-405. [CrossRef]

18. Khodaei, A. Resiliency-oriented microgrid optimal scheduling. IEEE Trans. Smart Grid 2014, 5, $1584-1591$. [CrossRef]

19. Hussain, A.; Bui, V.H.; Kim, H.M. Robust optimization-based scheduling of multi-microgrids considering uncertainties. Energies 2016, 9, 278. [CrossRef]

20. Gholami, A.; Shekari, T.; Aminifar, F.; Shahidehpour, M. Microgrid Scheduling With Uncertainty: The Quest for Resilience. IEEE Trans. Smart Grid 2016, 7, 2849-2858. [CrossRef]

21. Zakariazadeh, A.; Jadid, S.; Siano, P. Smart microgrid energy and reserve scheduling with demand response using stochastic optimization. Int. J. Electr. Power Energy Syst. 2014, 63, 523-533. [CrossRef]

22. Jayantilal, A.; McCarthy, C.A. Reducing Customer Outages-Smart Distribution through Layered Intelligence. NEMA Electroind. Mag. 2012, 3, 1170-1180

23. Che, L.; Khodayar, M.; Shahidehpour, M. Only connect: Microgrids for distribution system restoration. IEEE Power Energy Mag. 2014, 12, 70-81.

24. Gao, H.; Chen, Y.; Xu, Y.; Liu, C.C. Resilience-oriented critical load restoration using microgrids in distribution systems. IEEE Trans. Smart Grid 2016, 7, 2837-2848. [CrossRef]

25. Chen, C.; Wang, J.; Qiu, F.; Zhao, D. Resilient distribution system by microgrids formation after natural disasters. IEEE Trans. Smart Grid 2016, 7, 958-966. [CrossRef]

26. Kimble, S.J.; Vedullapalli, D.T.; Makram, E.B. Optimal Partitioning of Distribution Networks for Micro-Grid Operation. J. Power Energy Eng. 2017, 5, 104-120. [CrossRef]

27. Khodaei, A. Provisional microgrids. IEEE Trans. Smart Grid 2015, 6, 1107-1115. [CrossRef]

28. Gazijahani, F.S.; Ravadanegh, S.N.; Salehi, J. Stochastic multi-objective model for optimal energy exchange optimization of networked microgrids with presence of renewable generation under risk-based strategies. ISA Trans. 2018, 73, 100-111. [CrossRef] [PubMed]

29. Hatziargyriou, N.; Asano, H.; Iravani, R.; Marnay, C. Microgrids. IEEE Power Energy Mag. 2007, 5, 78-94. [CrossRef]

30. Kroposki, B.; Lasseter, R.; Ise, T.; Morozumi, S.; Papathanassiou, S.; Hatziargyriou, N. Making microgrids work. IEEE Power Energy Mag. 2008, 6, 40-53. [CrossRef]

31. Lidula, N.; Rajapakse, A. Microgrids research: A review of experimental microgrids and test systems. Renew. Sustain. Energy Rev. 2011, 15, 186-202. [CrossRef]

32. Olivares, D.E.; Mehrizi-Sani, A.; Etemadi, A.H.; Cañizares, C.A.; Iravani, R.; Kazerani, M.; Hajimiragha, A.H.; Gomis-Bellmunt, O.; Saeedifard, M.; Palma-Behnke, R.; et al. Trends in Microgrid Control. IEEE Trans. Smart Grid 2014, 5, 1905-1919. [CrossRef]

33. Nosratabadi, S.M.; Hooshmand, R.A.; Gholipour, E. A comprehensive review on microgrid and virtual power plant concepts employed for distributed energy resources scheduling in power systems. Renew. Sustain. Energy Rev. 2017, 67, 341-363. [CrossRef]

34. Han, Y.; Zhang, K.; Li, H.; Coelho, E.A.A.; Guerrero, J.M. MAS-based distributed coordinated control and optimization in microgrid and microgrid clusters: A comprehensive overview. IEEE Trans. Power Electron. 2017, 33, 6488-6508. [CrossRef]

35. Cheng, Z.; Duan, J.; Chow, M.Y. To centralize or to distribute: That is the question: A comparison of advanced microgrid management systems. IEEE Ind. Electron. Mag. 2018, 12, 6-24. [CrossRef] 
36. Hirsch, A.; Parag, Y.; Guerrero, J. Microgrids: A review of technologies, key drivers, and outstanding issues. Renew. Sustain. Energy Rev. 2018, 90, 402-411. [CrossRef]

37. Zia, M.F.; Elbouchikhi, E.; Benbouzid, M. Microgrids energy management systems: A critical review on methods, solutions, and prospects. Appl. Energy 2018, 222, 1033-1055. [CrossRef]

38. Chi, Y.; Xu, Y. Resilience-oriented microgrids: A comprehensive literature review. In Proceedings of the 2017 IEEE Innovative Smart Grid Technologies-Asia (ISGT-Asia), Auckland, New Zealand, 4-7 December 2017.

39. Feng, W.; Jin, M.; Liu, X.; Bao, Y.; Marnay, C.; Yao, C.; Yu, J. A review of microgrid development in the United States-A decade of progress on policies, demonstrations, controls, and software tools. Appl. Energy 2018, 228, 1656-1668. [CrossRef]

40. Alam, M.N.; Chakrabarti, S.; Ghosh, A. Networked microgrids: State-of-the-art and future perspectives. IEEE Trans. Ind. Informatics 2018, 15, 1238-1250. [CrossRef]

41. Boqtob, O.; El Moussaoui, H.; El Markhi, H.; Lamhamdi, T. Microgrid energy management system: A state-of-the-art review. J. Electr. Syst. 2019, 15, 53-67.

42. Wang, Y.; Chen, C.; Wang, J.; Baldick, R. Research on resilience of power systems under natural disasters-A review. IEEE Trans. Power Syst. 2015, 31, 1604-1613.

43. Lin, Y.; Bie, Z.; Qiu, A. A review of key strategies in realizing power system resilience. Glob. Energy Interconnect. 2018, 1, 70-78.

44. Anderson, A.A.; Suryanarayanan, S. Review of Energy Management and Planning of Islanded Microgrids. CSEE J. Power Energy Syst. 2019, 6, 329-343.

45. Moher, D.; Liberati, A.; Tetzlaff, J.; Altman, D.G.; Group, T.P. Preferred Reporting Items for Systematic Reviews and Meta-Analyses: The PRISMA Statement. PLoS Med. 2009, 6, e1000097. [CrossRef]

46. Spiegel, M.H. Poster Abstract: Towards Advanced Resiliency-Oriented Multi-Microgrid Scheduling. In Proceedings of the Abstr. 8th DACH+ Conf. Energy Informatics, Salzburg, Austria, 26-27 September 2019; Volume 2.

47. Farag, H.E.; Abdelaziz, M.M.A.; El-Saadany, E.F. Voltage and reactive power impacts on successful operation of islanded microgrids. IEEE Trans. Power Syst. 2013, 28, 1716-1727.

48. Khodayar, M.E.; Barati, M.; Shahidehpour, M. Integration of high reliability distribution system in microgrid operation. IEEE Trans. Smart Grid 2012, 3, 1997-2006. [CrossRef]

49. Bashiri, M. Optimal scheduling of distributed energy resources in a distribution system based on imperialist competitive algorithm considering reliability worth. Neural Comput. Appl. 2014, 25, 967-974. [CrossRef]

50. Kavousi-Fard, A.; Khodaei, A. Efficient integration of plug-in electric vehicles via reconfigurable microgrids. Energy 2016, 111, 653-663. [CrossRef]

51. Farzin, H.; Fotuhi-Firuzabad, M.; Moeini-Aghtaie, M. A stochastic multi-objective framework for optimal scheduling of energy storage systems in microgrids. IEEE Trans. Smart Grid 2017, 8, 117-127. [CrossRef]

52. Hosseinnezhad, V.; Rafiee, M.; Ahmadian, M.; Siano, P. Optimal day-ahead operational planning of microgrids. Energy Convers. Manag. 2016, 126, 142-157. [CrossRef]

53. Gholami, A.; Shekari, T.; Grijalva, S. Proactive management of microgrids for resiliency enhancement: An adaptive robust approach. IEEE Trans. Sustain. Energy 2017, 10, 470-480. [CrossRef]

54. Qiu, H.; Zhao, B.; Gu, W.; Bo, R. Bi-level two-stage robust optimal scheduling for AC/DC hybrid multi-microgrids. IEEE Trans. Smart Grid 2018, 9, 5455-5466. [CrossRef]

55. Gazijahani, F.S.; Salehi, J. Integrated DR and reconfiguration scheduling for optimal operation of microgrids using Hong's point estimate method. Int. J. Electr. Power Energy Syst. 2018, 99, 481-492. [CrossRef]

56. Kavousi-Fard, A.; Zare, A.; Khodaei, A. Effective dynamic scheduling of reconfigurable microgrids. IEEE Trans. Power Syst. 2018, 33, 5519-5530. [CrossRef]

57. Sarfi, V.; Livani, H. An economic-reliability security-constrained optimal dispatch for microgrids. IEEE Trans. Power Syst. 2018, 33, 6777-6786.

58. Salyani, P.; Ravadanegh, S.N.; Tabatabaei, N.M. Optimal Scheduling of Networked-Microgrids to Resiliency Enhancement Under Uncertainty. In Power Systems Resilience; Mahdavi Tabatabaei, N., Najafi Ravadanegh, S., Bizon, N., Eds.; Springer: Cham, Switzerland, 2019; Chapter 6.

59. Gan, L.K.; Hussain, A.; Howey, D.A.; Kim, H.M. Limitations in Energy Management Systems: A Case Study for Resilient Interconnected Microgrids. IEEE Trans. Smart Grid 2018, 10, 5675-5685. [CrossRef]

60. Yang, F.; Feng, X.; Li, Z. Advanced microgrid energy management system for future sustainable and resilient power grid. IEEE Trans. Ind. Appl. 2019, 55, 7251-7260. [CrossRef] 
61. Teimourzadeh, S.; Tor, O.B.; Cebeci, M.E.; Adela, B.; Oprea, S.V. A three-stage approach for resilienceconstrained scheduling of networked microgrids. J. Mod. Power Syst. Clean Energy 2019, 7, 705-715. [CrossRef]

62. Liang, Z.; Alsafasfeh, Q.; Su, W. Proactive Resilient Scheduling for Networked Microgrids With Extreme Events. IEEE Access 2019, 7, 112639-112652. [CrossRef]

63. Ananduta, W.; Maestre, J.M.; Ocampo-Martinez, C.; Ishii, H. Resilient distributed model predictive control for energy management of interconnected microgrids. Optim. Control Appl. Methods 2020, 41, 146-169. [CrossRef]

64. Zadsar, M.; Sebtahmadi, S.S.; Kazemi, M.; Larimi, S.; Haghifam, M. Two stage risk based decision making for operation of smart grid by optimal dynamic multi-microgrid. Int. J. Electr. Power Energy Syst. 2020, 118, 105791. [CrossRef]

65. Liu, N.; Chen, Q.; Liu, J.; Lu, X.; Li, P.; Lei, J.; Zhang, J. A heuristic operation strategy for commercial building microgrids containing EVs and PV system. IEEE Trans. Ind. Electron. 2014, 62, 2560-2570. [CrossRef]

66. Hussain, A.; Bui, V.H.; Kim, H.M. A proactive and survivability-constrained operation strategy for enhancing resilience of microgrids using energy storage system. IEEE Access 2018, 6, 75495-75507. [CrossRef]

67. Hussain, A.; Bui, V.H.; Kim, H.M. Resilience-oriented optimal operation of networked hybrid microgrids. IEEE Trans. Smart Grid 2019, 10, 204-215. [CrossRef]

68. Bornapour, M.; Hooshmand, R.A.; Khodabakhshian, A.; Parastegari, M. Optimal stochastic scheduling of CHP-PEMFC, WT, PV units and hydrogen storage in reconfigurable micro grids considering reliability enhancement. Energy Convers. Manag. 2017, 150, 725-741. [CrossRef]

69. Cardoso, G.; Stadler, M.; Siddiqui, A.; Marnay, C.; DeForest, N.; Barbosa-Póvoa, A.; Ferrão, P. Microgrid reliability modeling and battery scheduling using stochastic linear programming. Electr. Power Syst. Res. 2013, 103, 61-69. [CrossRef]

70. Conradsen, K.; Nielsen, L.; Prahm, L. Review of Weibull statistics for estimation of wind speed distributions. J. Clim. Appl. Meteorol. 1984, 23, 1173-1183. [CrossRef]

71. Emeis, S. Wind Energy Meteorology: Atmospheric Physics for Wind Power Generation; Springer: Cham, Switzerland, 2018.

72. Salameh, Z.M.; Borowy, B.S.; Amin, A.R. Photovoltaic module-site matching based on the capacity factors. IEEE Trans. Energy Convers. 1995, 10, 326-332. [CrossRef]

73. Blum, C.; Puchinger, J.; Raidl, G.R.; Roli, A. Hybrid metaheuristics in combinatorial optimization: A survey. Appl. Soft Comput. 2011, 11, 4135-4151. [CrossRef]

74. Gazijahani, F.S.; Salehi, J. Stochastic multi-objective framework for optimal dynamic planning of interconnected microgrids. IET Renew. Power Gener. 2017, 11, 1749-1759. [CrossRef]

75. Chis, M.; Salama, M.; Jayaram, S. Capacitor placement in distribution systems using heuristic search strategies. IEE Proc.-Gener. Transm. Distrib. 1997, 144, 225-230. [CrossRef]

76. Conti, S.; Nicolosi, R.; Rizzo, S.; Zeineldin, H. Optimal dispatching of distributed generators and storage systems for MV islanded microgrids. IEEE Trans. Power Deliv. 2012, 27, 1243-1251. [CrossRef]

77. Baran, M.E.; Wu, F.F. Network reconfiguration in distribution systems for loss reduction and load balancing. IEEE Trans. Power Deliv. 1989, 4, 101-102. [CrossRef]

78. New York Independent System Operator. Available online: https://www.nyiso.com/ (accessed on 14 October 2019).

79. Zolfaghari, S.; Riahy, G.H.; Abedi, M. A new method to adequate assessment of wind farms' power output. Energy Convers. Manag. 2015, 103, 585-604. [CrossRef]

80. Nguyen, D.T.; Le, L.B. Optimal bidding strategy for microgrids considering renewable energy and building thermal dynamics. IEEE Trans. Smart Grid 2014, 5, 1608-1620. [CrossRef]

81. Khodaei, A. Microgrid optimal scheduling with multi-period islanding constraints. IEEE Trans. Power Syst. 2014, 29, 1383-1392.

82. McKenna, E.; Thomson, M. High-resolution stochastic integrated thermal-electrical domestic demand model. Appl. Energy 2016, 165, 445-461. [CrossRef]

83. NREL Measurement and Instrumentation Data Center. Available online: https://midcdmz.nrel.gov/ (accessed on 12 May 2020).

84. Turkish Energy Exchange Platform (EXIST). Available online: https://seffaflik.epias.com.tr/transparency/ (accessed on 16 April 2020).

85. Wang, J.; Shahidehpour, M.; Li, Z. Security-constrained unit commitment with volatile wind power generation. IEEE Trans. Power Syst. 2008, 23, 1319-1327. [CrossRef] 
86. PJM Interconnection-Markets and Operation. Available online: https://www.pjm.com/markets-andoperations.aspx (accessed on 12 May 2020).

87. Jager, D.; Andreas, A. NREL National Wind Technology Center (NWTC): M2 Tower; Boulder, Colorado (Data); Technical Report NREL/DA-5500-56489; National Renewable Energy Lab. (NREL): Golden, CO, USA, 1996.

88. Liang, Z.; Bian, D.; Zhang, X.; Shi, D.; Diao, R.; Wang, Z. Optimal energy management for commercial buildings considering comprehensive comfort levels in a retail electricity market. Appl. Energy 2019, 236, 916-926. [CrossRef]

89. Weniger, J.; Maier, S.; Orth, N.; Quaschning, V. Stromspeicher-Inspektion 2020; Technical report; Hochschule für Technik und Wirtschaft: Berlin, Germany, 2020.

90. Schimpe, M.; Naumann, M.; Truong, N.; Hesse, H.C.; Santhanagopalan, S.; Saxon, A.; Jossen, A. Energy efficiency evaluation of a stationary lithium-ion battery container storage system via electro-thermal modeling and detailed component analysis. Appl. Energy 2018, 210, 211-229. [CrossRef]

91. Bludszuweit, H.; Domínguez-Navarro, J.A.; Llombart, A. Statistical analysis of wind power forecast error. IEEE Trans. Power Syst. 2008, 23, 983-991. [CrossRef]

92. Clarke, D.; Gawthrop, P. Self-tuning control. In Proceedings of the Institution of Electrical Engineers; IET: London, UK, 1979.

93. Dou, X.; Xu, P.; Hu, Q.; Sheng, W.; Quan, X.; Wu, Z.; Xu, B. A distributed voltage control strategy for multi-microgrid active distribution networks considering economy and response speed. IEEE Access 2018, 6, 31259-31268. [CrossRef]

94. Russell, S.J.; Norvig, P.V. Artificial Intelligence, 4th ed.; Pearson: Boston, MA, USA, 2019.

95. Andrén, F.P.; Strasser, T.I.; Kastner, W. Engineering smart grids: Applying model-driven development from use case design to deployment. Energies 2017, 10, 374. [CrossRef]

96. Parag, Y.; Sovacool, B.K. Electricity market design for the prosumer era. Nat. Energy 2016, 1, 1-6. [CrossRef]

97. Kavousi-Fard, A.; Wang, M.; Su, W. Stochastic resilient post-hurricane power system recovery based on mobile emergency resources and reconfigurable networked microgrids. IEEE Access 2018, 6, 72311-72326. [CrossRef]

98. Spalthoff, C.; Sarajlić, D.; Kittl, C.; Drauz, S.; Kneiske, T.; Rehtanz, C.; Braun, M. SimBench: Open source time series of power load, storage and generation for the simulation of electrical distribution grids. In Proceedings of the Internationaler ETG-Kongress, Preprint, Esslingen, Germany, 8-9 May 2019.

99. Meinecke, S.; Bornhorst, N.; Braun, M. Power system benchmark generation methodology. In Proceedings of the NEIS 2018, Conference on Sustainable Energy Supply and Energy Storage Systems, Hamburg, Germany, 20-21 September 2018.

100. Schneider, K.; Mather, B.; Pal, B.; Ten, C.W.; Shirek, G.; Zhu, H.; Fuller, J.; Pereira, J.; Ochoa, L.; De Araujo, L.; et al. Analytic considerations and design basis for the IEEE distribution test feeders. IEEE Trans. Power Syst. 2017, 33, 3181-3188. [CrossRef]

101. Heussen, K.; Steinbrink, C.; Abdulhadi, I.F.; Nguyen, V.H.; Degefa, M.Z.; Merino, J.; Jensen, T.V.; Guo, H.; Gehrke, O.; Bondy, D.E.M.; et al. ERIGrid holistic test description for validating cyber-physical energy systems. Energies 2019, 12, 2722. [CrossRef]

102. Ebe, F.; Idlbi, B.; Casel, M.; Kondzialka, C.; Heilscher, G.; Seitl, C.; Bruendlinger, R.; Strasser, T. An approach for validating and testing micro grid and cell-based control concepts. In Proceedings of the CIRED 2018 Ljubljana Workshop on Microgrids and Local Energy Communities, Ljubljana, Slovenia, 7-8 June 2018.

103. Strasser, T.I.; de Jong, E.C.; Sosnina, M. (Eds.) European Guide to Power System Testing; Springer: Cham, Switzerland, 2020.

(C) 2020 by the authors. Licensee MDPI, Basel, Switzerland. This article is an open access article distributed under the terms and conditions of the Creative Commons Attribution (CC BY) license (http:// creativecommons.org/licenses/by/4.0/). 